\title{
WestVirginiaUniversity
}

THE RESEARCH REPOSITORY @ WVU

Graduate Theses, Dissertations, and Problem Reports

2018

\section{An Analysis of the Extinction-Induced Response Burst}

Brian Katz

Follow this and additional works at: https://researchrepository.wvu.edu/etd

\section{Recommended Citation}

Katz, Brian, "An Analysis of the Extinction-Induced Response Burst" (2018). Graduate Theses, Dissertations, and Problem Reports. 5943.

https://researchrepository.wvu.edu/etd/5943

This Thesis is protected by copyright and/or related rights. It has been brought to you by the The Research Repository @ WVU with permission from the rights-holder(s). You are free to use this Thesis in any way that is permitted by the copyright and related rights legislation that applies to your use. For other uses you must obtain permission from the rights-holder(s) directly, unless additional rights are indicated by a Creative Commons license in the record and/ or on the work itself. This Thesis has been accepted for inclusion in WVU Graduate Theses, Dissertations, and Problem Reports collection by an authorized administrator of The Research Repository @ WVU. For more information, please contact researchrepository@mail.wvu.edu. 
An Analysis of the Extinction-Induced Response Burst

Brian Katz

\author{
Thesis submitted \\ to the Eberly College of Arts and Sciences \\ at West Virginia University \\ in partial fulfillment of the requirements for the degree of \\ Master of Science in \\ Psychology
}

\title{
Kennon A. Lattal, Ph.D., Chair \\ Michael Perone, Ph.D. \\ Barry Edelstein, Ph.D. \\ Department of Psychology
}

Morgantown, West Virginia

2018

Keywords: extinction, extinction burst, repeated extinction, pigeons

Copyright 2018 Brian Katz 


\section{ABSTRACT \\ An Analysis of the Extinction-Induced Response Burst}

\section{Brian Katz}

Although the extinction burst is a frequently reported generative effect of extinction, there are few experimental analyses of the phenomenon. The purpose of the present series of experiments was to examine the occurrence, time course, and repeatability of extinction bursts. Six experimentally naïve pigeons were exposed to at least five cycles of 5-sessions block of baseline followed by 8 -session blocks of extinction. Depending on the condition, baseline sessions were either a fixed-ratio (FR) or variable-ratio (VR) schedule, and transitions from the last baseline session in each cycle to the first extinction session were conducted either between or within sessions. Within a block, subsequent extinction sessions were in effect throughout the session. There was not a single instance of an extinction burst when whole-session response rates were considered. Restricting the analysis to the first minute of an extinction session sometimes revealed a burst, most often during the first extinction session of a block, although this finding was not consistent. The frequency and magnitude of the extinction burst differed across exposures to extinction both across and within pigeons. Additionally, details of how the burst was measured (i.e. the level of analysis and definition of the phenomenon) influenced the occurrence and dimensions of the extinction burst. The results of the three experiments suggest that the way in which extinction is implemented and how the burst is defined influence whether or not a burst-like increase in responding is observed at the onset of extinction. Under the best of conditions, the extinction burst does not appear to be a reliable generative effect of extinction.

Keywords: extinction, extinction burst, repeated extinction, pigeons 


\section{Acknowledgements}

I would like to thank Andy Lattal for his mentorship and assistance with designing these experiments, scientific writing, and data interpretation. Without his help this thesis would not have been possible. I also would like to thank the members of my thesis committee, Andy Lattal, Mike Perone, and Barry Edelstein, who provided many helpful comments and suggestions concerning these experiments. I also am grateful to the members of the Lattal Lab for their help with both taking care of the animals and conducting experimental sessions. Additionally, I would like to thank Forrest Toegel for his opinions on the nature of the extinction eruption. Finally, I would like to thank my parents for their support and words of wisdom throughout this

process. Both were always eager to hear about the current state of these experiments, and did not hesitate to offer their own interpretations and suggestions concerning the latest results. 


\section{Table of Contents}

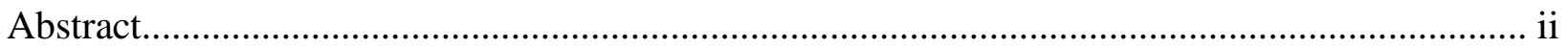

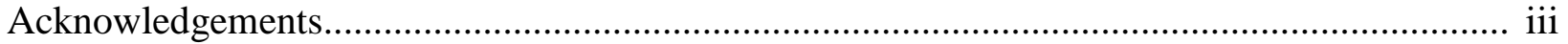

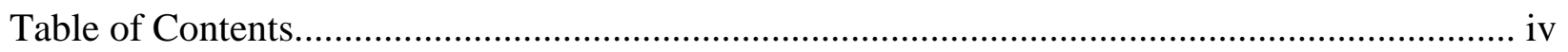

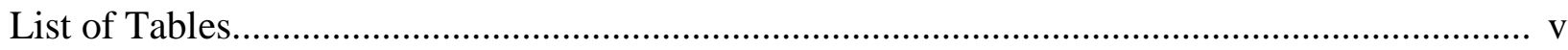

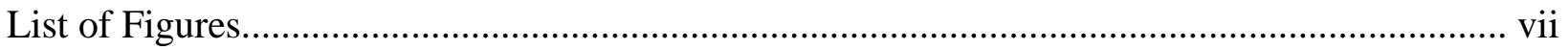

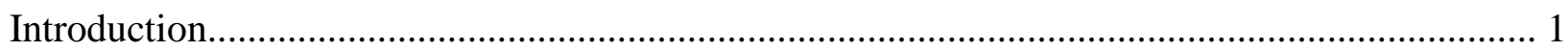

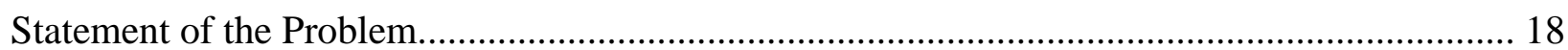

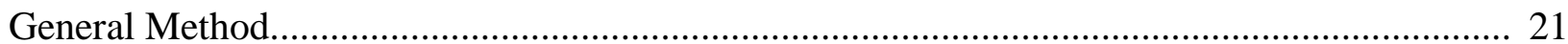

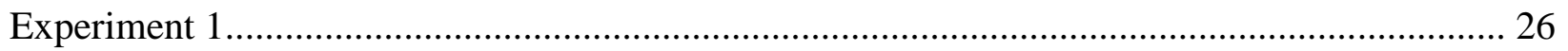

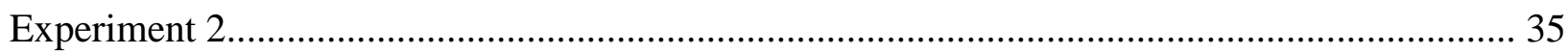

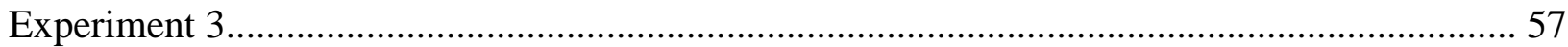

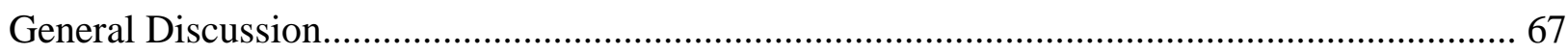

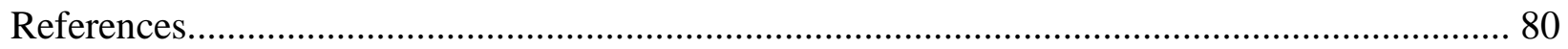




\section{List of Tables}

Table 1. Pigeons used in each of the three experiments.....

Table 2. Comparison of response rates in the first session of each block of extinction to each of the three criteria for bursting. Y's indicate that the criterion for bursting was met, whereas N's indicate that it was not. Numbers in parentheses indicate first-extinction-session response rates calculated as a percentage of the indicated baseline criterion and rounded to the nearest whole number. For comparisons to the mean or final session, percents are only included if the criterion was met. For comparisons to the maximum, percents are included if the criterion was met or if either of the previous criteria were met. A dashed line indicates that a percent could not be calculated for that comparison.

Table 3. Sequence, type, and number of transitions for each pigeon in Experiment 2

Table 4. Comparison of response rates in the first session of each block of extinction (betweensessions transitions) to each of the three criteria for bursting. Y's indicate that the criterion for bursting was met, whereas N's indicate that it was not. Numbers in parentheses indicate firstextinction-session response rates calculated as a percentage of the indicated baseline criterion and rounded to the nearest whole number. For comparisons to the mean or final session, percents are only included if the criterion was met. For comparisons to the maximum, percents are included if the criterion was met or if either of the previous criteria were met. A dashed line indicates that a percent could not be calculated for that comparison.

Table 5. Comparison of first-minute response rates from the first session of each block of extinction (within-session transitions cf. start of baseline) to each of the three criteria for bursting. Y's indicate that the criterion for bursting was met, whereas N's indicate that it was not. Numbers in parentheses indicate first-extinction-session response rates calculated as a percentage of the indicated baseline criterion and rounded to the nearest whole number. For comparisons to the mean or final session, percents are only included if the criterion was met. For comparisons to the maximum, percents are included if the criterion was met or if either of the previous criteria were met.

Table 6. Comparison of first-minute response rates from the first session of each block of extinction (within-session transitions cf. end of baseline) to each of the three criteria for bursting. Y's indicate that the criterion for bursting was met, whereas N's indicate that it was not. Numbers in parentheses indicate first-extinction-session response rates calculated as a percentage of the indicated baseline criterion and rounded to the nearest whole number. For comparisons to the mean or final session, percents are only included if the criterion was met. For comparisons to the maximum, percents are included if the criterion was met or if either of the previous criteria were met. 
Table 7. Comparison of both first-minute response rates and first-minute run rates from the first session of each block of extinction (within-session transitions cf. start of baseline) to each of the three criteria for bursting. Y's indicate that the criterion for bursting was met, whereas N's indicate that it was not. Numbers in parentheses indicate first-extinction-session response rates or run rates calculated as a percentage of the indicated baseline criterion and rounded to the nearest whole number. For comparisons to the mean or final session, percents are only included if the criterion was met. For comparisons to the maximum, percents are included if the criterion was met or if either of the previous criteria were met.

Table 8. Comparison of both first-minute response rates and first-minute run rates from the first session of each block of extinction (within-session transitions cf. end of baseline) to each of the three criteria for bursting. Y's indicate that the criterion for bursting was met, whereas N's indicate that it was not. Numbers in parentheses indicate first-extinction-session response rates or run rates calculated as a percentage of the indicated baseline criterion and rounded to the nearest whole number. For comparisons to the mean or final session, percents are only included if the criterion was met. For comparisons to the maximum, percents are included if the criterion was met or if either of the previous criteria were met. 52

Table 9. Number of Transitions For Each Pigeon in Experiment 3. 58

Table 10. Comparison of first-minute response rates from the first session of each block of extinction (within-session transitions cf. start of baseline) to each of the three criteria for bursting. Y's indicate that the criterion for bursting was met, whereas N's indicate that it was not. Numbers in parentheses indicate first-extinction-session response rates calculated as a percentage of the indicated baseline criterion and rounded to the nearest whole number. For comparisons to the mean or final session, percents are only included if the criterion was met. For comparisons to the maximum, percents are included if the criterion was met or if either of the previous criteria were met.

Table 11. Comparison of first-minute response rates from the first session of each block of extinction (within-session transitions cf. end of baseline) to each of the three criteria for bursting. Y's indicate that the criterion for bursting was met, whereas N's indicate that it was not.

Numbers in parentheses indicate first-extinction-session response rates calculated as a percentage of the indicated baseline criterion and rounded to the nearest whole number. For comparisons to the mean or final session, percents are only included if the criterion was met. For comparisons to the maximum, percents are included if the criterion was met or if either of the previous criteria were met. 


\section{List of Figures}

Figure 1. Response rates for each pigeon across blocks of the repeated-extinctions procedure at the whole-session level of analysis. The upper panel shows absolute response rates per minute for the sessions shown. The lower panel shows the upper-panel data transformed to a percentage of the mean response rate of each block of Conditioning Phase 2. Solid horizontal lines in Conditioning Phase 2 sections of the lower panel show the mean baseline response rate. Data points superimposed over this line are the response rates for the final session of Conditioning Phase 2 calculated as a percentage of the mean response rate for the phase. In those blocks containing fewer than five (Conditioning Phase 2) or eight (Extinction phase) data points, technical problems resulted in lost data for those sessions. Note that only the first baseline (BSL) is identified. All of the panels without labels are subsequent baseline conditions.....

Figure 2. Response rates for each pigeon across blocks of the repeated-extinction procedure at the first-minute level of analysis. The upper panel shows the absolute response rate per minute for the sessions shown. The lower panel shows the upper-panel data transformed to a percentage of the mean response rate of each block of Conditioning Phase 2. Solid horizontal lines in Conditioning Phase 2 sections of the lower panel show the mean baseline response rate. Data points superimposed over this line are the response rates for the final session of Conditioning Phase 2 calculated as a percentage of the mean response rate for the phase. In those blocks containing fewer than five (Conditioning Phase 2) or eight (Extinction phase) data points, technical problems resulted in lost data for those sessions. Note that only the first baseline (BSL) is identified. All of the panels without labels are subsequent baseline conditions.....

Figure 3. Response rates for both pigeons across all blocks of between-sessions transitions at the whole-session level of analysis. The upper panel shows the absolute response rate per minute for the sessions shown. The lower panel shows the upper-panel data transformed to a percentage of the mean response rate of each block of Conditioning Phase 2. Solid horizontal lines in Conditioning Phase 2 sections of the lower panel show the mean baseline response rate. Data points superimposed over this line are the response rates for the final session of Conditioning Phase 2 calculated as a percentage of the mean response rate for the phase. In those blocks containing fewer than five (Conditioning Phase 2) or eight (Extinction phase) data points, technical problems resulted in lost data for those sessions. Note that only the first baseline (VR)

is identified. All of the panels without labels are subsequent baseline conditions

Figure 4. Response rates for both pigeons across all blocks of between-sessions transitions at the first-minute level of analysis. The upper panel shows the absolute response rate per minute for the sessions shown. The lower panel shows the upper-panel data transformed to a percentage of the mean response rate of each block of Conditioning Phase 2. Solid horizontal lines in Conditioning Phase 2 sections of the lower panel show the mean baseline response rate. Data points superimposed over this line are the response rates for the final session of Conditioning Phase 2 calculated as a percentage of the mean response rate for the phase. In those blocks containing fewer than five (Conditioning Phase 2) or eight (Extinction phase) data points, technical problems resulted in lost data for those sessions. Note that only the first baseline (VR) is identified. All of the panels without labels are subsequent baseline conditions 
Figure 5. Response rates for each pigeon across all blocks of within-session transitions at the whole-session level of analysis. The upper panel shows the absolute response rate per minute for the sessions shown. The lower panel shows the upper-panel data transformed to a percentage of the mean response rate of each block of Conditioning Phase 2. Solid horizontal lines in Conditioning Phase 2 sections of the lower panel show the mean baseline response rate. Data points superimposed over this line are the response rates for the final session of Conditioning Phase 2 calculated as a percentage of the mean response rate for the phase. In those blocks containing fewer than five (Conditioning Phase 2) or eight (Extinction phase) data points, technical problems resulted in lost data for those sessions. Note that only the first baseline (VR) is identified. All of the panels without labels are subsequent baseline conditions

Figure 6. Response rates for each pigeon across all blocks of within-session transitions at the first-minute level of analysis cf. the start of Conditioning Phase 2 sessions. The upper panel shows the absolute response rate per minute for the sessions shown. The lower panel shows the upper-panel data transformed to a percentage of the mean response rate of each block of Conditioning Phase 2. Solid horizontal lines in Conditioning Phase 2 sections of the lower panel show the mean baseline response rate. Data points superimposed over this line are the response rates for the final session of Conditioning Phase 2 calculated as a percentage of the mean response rate for the phase. In those blocks containing fewer than five (Conditioning Phase 2) or eight (Extinction phase) data points, technical problems resulted in lost data for those sessions. Note that only the first baseline (VR) is identified. All of the panels without labels are subsequent baseline conditions......

Figure 7. Response rates for each pigeon across all blocks of within-session transitions at the first-minute level of analysis cf. the end of Conditioning Phase 2 sessions. The upper panel shows the absolute response rate per minute for the sessions shown. The lower panel shows the upper-panel data transformed to a percentage of the mean response rate of each block of Conditioning Phase 2. Solid horizontal lines in Conditioning Phase 2 sections of the lower panel show the mean baseline response rate. Data points superimposed over this line are the response rates for the final session of Conditioning Phase 2 calculated as a percentage of the mean response rate for the phase. In those blocks containing fewer than five (Conditioning Phase 2) or eight (Extinction phase) data points, technical problems resulted in lost data for those sessions. Note that only the first baseline (VR) is identified. All of the panels without labels are subsequent baseline conditions.

Figure 8. Response rates for each pigeon across blocks of the repeated-extinction procedure at the whole-session level of analysis. The upper panel shows the absolute response rate per minute for the sessions shown. The lower panel shows the upper-panel data transformed to a percentage of the mean response rate of each block of Conditioning Phase 2. Solid horizontal lines in Conditioning Phase 2 sections of the lower panel show the mean baseline response rate. Data points superimposed over this line are the response rates for the final session of Conditioning Phase 2 calculated as a percentage of the mean response rate for the phase. In those blocks containing fewer than five (Conditioning Phase 2) or eight (Extinction phase) data points, technical problems resulted in lost data for those sessions. Note that only the first baseline (FR) is identified. All of the panels without labels are subsequent baseline conditions. 
Figure 9. Response rates for each pigeon across all blocks of the repeated-extinction procedure at the first-minute level of analysis cf. the start of Conditioning Phase 2 sessions. The upper panel shows the absolute response rate per minute for the sessions shown. The lower panel shows the upper-panel data transformed to a percentage of the mean response rate of each block of Conditioning Phase 2. Solid horizontal lines in Conditioning Phase 2 sections of the lower panel show the mean baseline response rate. Data points superimposed over this line are the response rates for the final session of Conditioning Phase 2 calculated as a percentage of the mean response rate for the phase. In those blocks containing fewer than five (Conditioning Phase 2) or eight (Extinction phase) data points, technical problems resulted in lost data for those sessions. Note that only the first baseline (FR) is identified. All of the panels without labels are subsequent baseline conditions

Figure 10. Response rates for each pigeon across all blocks of the repeated-extinction procedure at the first-minute level of analysis cf. the end of Conditioning Phase 2 sessions. The upper panel shows the absolute response rate per minute for the sessions shown. The lower panel shows the upper-panel data transformed to a percentage of the mean response rate of each block of Conditioning Phase 2. Solid horizontal lines in Conditioning Phase 2 sections of the lower panel show the mean baseline response rate. Data points superimposed over this line are the response rates for the final session of Conditioning Phase 2 calculated as a percentage of the mean response rate for the phase. In those blocks containing fewer than five (Conditioning Phase 2) or eight (Extinction phase) data points, technical problems resulted in lost data for those sessions. Note that only the first baseline (FR) is identified. All of the panels without labels are subsequent baseline conditions

Figure 11. A comparison of an extinction burst (left panel) and an extinction eruption (right panel) obtained during two different transitions to extinction in Experiment 2. Note how much more intense the right panel looks. 
Contingencies of reinforcement and punishment, like apparatuses, "sometimes break down" (Skinner, 1956/1961, p. 109). Such breakdowns may occur through experimenter error, degradation, transformation, relaxation, or intentional removal of these contingencies. Although much of the experimental analysis has been concerned with how intact contingencies maintain behavior, the effects of the aforementioned changes also are of interest. These changes have been studied in various ways, but one of the most widely studied, and perhaps best understood, is extinction.

Extinction is defined herein as the reduction or elimination of responding when a response that was previously reinforced is no longer followed by the reinforcer. Extinction typically culminates in the rate of the previously reinforced behavior declining to baseline levels, often zero. As the rate of the target response declines, new responses often emerge, metaphorically replacing the target response. This phenomenon is referred to as the generative effect of extinction (e.g., Lattal, St. Peter, \& Escobar, 2013). One such generative effect of extinction reportedly is the extinction-induced response burst (hereafter, response burst, extinction burst, or burst), a phenomenon in which, "the frequency of responding may temporarily increase before beginning its decline" (Reynolds, 1968, p. 30).

Little attention has been given to defining the extinction burst with precision, let alone systematically identifying the variables that control it. Because the extinction burst is inextricably linked to the extinction process itself, the following literature review first considers the variables controlling extinction and then focuses more specifically on experimental investigations of the extinction burst. This review is followed by a proposal of research designed to examine the extinction burst, its repeatability over successive exposures to extinction, and some of its controlling variables. 


\section{Literature Review}

\section{Eliminative Effects of Extinction}

When the controlling variables of a given response disappear, the frequency or rate of that response will change. In the case of a response maintained by either positive or negative reinforcement, removal of reinforcement either reduces or completely eliminates responding. This phenomenon is extinction. Extinction can be implemented for responses that are either positively or negatively reinforced; however, the current discussion will focus on the extinction of responses previously maintained by positive reinforcement.

For these latter responses, extinction can be implemented in two ways. First, as described above, the positive reinforcers can be removed such that responses that previously resulted in the delivery of the reinforcer no longer do so. This procedure has been widely replicated and reliably shown to eliminate responding, at least while the extinction procedure is in effect (Lattal, 1972; Skinner, 1938). Extinction also can be implemented by removing the response-reinforcer dependency. Like the removal of the positive reinforcer, eliminating the response-reinforcer dependency also consistently reduces, but may or may not eliminate responding (Appel \& Hiss, 1962; Edwards, Peek, \& Wolfe, 1970; Lattal \& Maxey, 1971; Rescorla \& Skucy, 1969; Zeiler, 1968). Although both procedures eliminate responding, removing the reinforcer reduces responding faster and often to lower levels than does removing the response-reinforcer dependency (Lattal, 1972; Rescorla \& Skucy, 1969). As the proposed experiment will involve the rapid elimination of responding, the current discussion will focus on conventional extinction.

The main effect of extinction, whether it is implemented via the removal of a positive reinforcer or the elimination of a response-reinforcer dependency, is a reduction in response rate. 
The time course of extinction, however, is influenced by properties of the reinforcement schedule that maintained the target response. Variables such as the frequency, magnitude, delay, and type of reinforcement schedule all influence the course of extinction (e.g., Nevin, 1974;

Reynolds, 1968). Specifically, responses maintained by schedules that produce more frequent delivery of reinforcers, longer-duration reinforcers, or shorter delays to reinforcement are more resistant to extinction than those maintained by less frequent, smaller, or more delayed reinforcers. Additionally, schedule requirements of a specific response rate can affect the characteristics of extinction. In one experiment, for example, schedules that reinforced lower response rates (differential-reinforcement-of-low-rate schedules) were more resistant to extinction than those that reinforced high response rates (differential reinforcement of high-rate behavior) (Nevin, 1974).

Properties of the extinction procedure itself also can influence the course of extinction. One such property is the number of successive cycles of reinforcement of a response followed by its extinction. Using pigeons as subjects, Anger and Anger (1976) alternated eight successive 43-min extinction sessions with two-day conditioning sessions. During each conditioning session, responses were reinforced according to a variable-ratio (VR) schedule in which the ratio requirement progressively increased from a VR 1 to a VR 5 over the course of 45 reinforcements. After conditioning sessions, responding was extinguished by eliminating the delivery of reinforcement for responding. During the first block of extinction, response rates were considerably higher during the first extinction session compared to the remaining seven days. The magnitude of this difference decreased over successive blocks of extinction: by the sixth extinction period, response rates were greater than zero and about the same during each of 
the eight extinction sessions. Thus, the extent of the eliminative effect of extinction is modulated by the iteration of the procedure.

In a similar vein, Terrace (1963) found that the manner in which an extinction procedure is introduced influences the degree of behavior change. In conventional studies of extinction, including all those discussed herein thus far, the transition between conditioning and extinction is abrupt. In such procedures, in which a period of nonreinforcement suddenly replaces an existing schedule of reinforcement, many responses are made during extinction. Conversely, when extinction was introduced gradually by repeatedly alternating periods of reinforcement with progressively longer periods of nonreinforcement, fewer responses occurred during the extinction periods.

\section{Generative Effects of Extinction}

Decreases in response rate, despite being the most well-known effect of extinction, are not the only consequence. Although extinction procedures do eventually reduce the rate of the target response, the onset of extinction also can at least transiently increase the frequency of responses other than the one undergoing extinction. Such increases can be manifest in several ways, which are collectively referred to as the generative effects of extinction (Lattal et al., 2013). Three examples of such generative effects are increases in response variability, the emergence or recurrence of alternative behavior, and the extinction burst.

Extinction often increases the variability of response location (Antonitis, 1951; Eckerman \& Lanson, 1969), duration (Margulies, 1961), force (Notterman \& Mintz, 1965), topography (Stokes, 1995), and number (Mechner, 1958). Antonitis (1951), for example, placed rats in an operant chamber fitted with a 50-cm long aperture to which they could direct nose pokes. Pokes along any part of the long aperture were reinforced according to a fixed-ratio (FR) 1 schedule 
and triggered a camera to record the location of the poke. After reinforcing 225 nose pokes over 5 days, nose poking no longer was reinforced for four sessions, and any changes in variability (nose poke locations along the aperture) were observed. Early in conditioning, there was a great deal of variability in nose poke location along the aperture. Variability decreased over the course of conditioning, but then substantially increased again upon the transition to extinction. This greater variation, relative to that which occurred at the end of the training condition, persisted across the four extinction sessions.

Along with increases in the variability of the target response, introducing extinction also may promote the emergence of various alternative topographies to the one undergoing extinction (Azrin, Hutchinson, \& Hake, 1966; Kelly \& Hake, 1970; see also Epstein, 1983; Lattal \& St. Peter Pipkin, 2009). Much of the research concerning emergent alternative responses has centered on extinction-induced aggression. In such instances, the extinction of a previously reinforced response in the presence of an appropriate target for aggression leads to the subject engaging in various aggressive responses directed to that target (Azrin et al., 1966; Goh \& Iwata, 1994; Thompson \& Bloom, 1966). Aggression is only one of a variety of responses that can be induced by extinction. Others include polydipsia, that is, schedule induced drinking (Roper, 1981), wheel running (Staddon, 1977), and grooming (Staddon, 1977).

\section{Defining the Extinction Burst}

An early report of the course of extinction noted that an extinction curve usually, "begins with a steeper slope (higher response rate) than that during regular reinforcement...partly because the animal is apt to attack vigorously the now-unrewarding bar" (Keller \& Schoenfeld, 1950, p. 71). This short-lived, pronounced increase in response rate that occurs with the onset of extinction is labeled the extinction burst, and may be a third generative effect of extinction. 
Following this increase in response rate early during extinction, rates will decrease and eventually approach zero.

The extinction burst is most frequently described as a transient increase in response rate, but it also can manifest as a facilitation of other dimensions of a target response relative to their occurrence in the immediately preceding reinforcement condition. For example, Holton (1961) showed that the amplitude of a target response increased immediately following the onset of extinction. Children were taught to press against one of two windows to receive tokens that could be exchanged for prizes. Tokens were delivered on an FR 1 schedule, and 11 were required to earn a prize. After either 13 or 26 prizes were earned, tokens were withheld on four subsequent trials and response amplitude was measured. Subjects pressed the window significantly more forcefully (as measured by force exerted on the scale on which the windows were mounted) in these extinction trials than in the final four trials during baseline.

Furthermore, the onset of extinction can lead to a sudden increase in response duration. Margulies (1961) studied rats in a standard operant chamber, where they received food for lever pressing according to an FR 1 schedule. Lever-press duration on each trial was recorded by means of a microswitch that remained closed while the lever was pressed. After between 1 and 21 sessions, extinction replaced the FR 1 schedule. The duration of each lever press gradually declined over the course of conditioning, but immediately increased on the transition to extinction. This greater duration relative to that at the end of lever-press conditioning persisted throughout the five extinction sessions.

Although the extinction burst has been described as one of - if not the most - frequently reported generative effects of extinction (e.g., Ducharme \& Van Houten, 1994; Kazdin, 1994; Martin \& Pear, 1992), precise operational definitions of the phenomenon are few. In many 
textbooks and primers the extinction burst is discussed in very broad, general terms. Cooper, Heron, and Heward (2007), for example, defined the extinction burst as, "an immediate increase in the frequency of the response after the removal of the positive, negative, or automatic reinforcement" (p. 462); Domjan and Burkhard (1982) stated that during extinction, "the subject may respond rapidly at first and then gradually slow down" (p. 163); Reynolds (1968) simply noted that, "the frequency of responding may temporarily increase" (p. 30) after extinction begins; and Millenson (1967) remarked that one outcome of extinction is, "an increase in the variability of the form (topography) and of the magnitude of the response" (p. 104). These authors provide broad generalizations and statements concerning the overall course of extinction and the occurrence of the extinction burst without identifying controlling variables in the preceding reinforcement condition; specific, empirical characteristics of the phenomenon; or specific experiments in which it has been found.

A few other definitions of the extinction burst have approached more precise descriptions of the phenomenon. Lerman and Iwata (1995, p. 93), for example, defined the extinction burst as, "an increase in responding during any of the first three treatment sessions above that observed during all of the last five baseline sessions (or all of baseline if it was briefer than five sessions)." In contrast to the rather general definitions offered in the previous paragraph, Lerman and Iwata at least delineated two important qualities of an extinction burst: (1) a specific period during extinction in which a burst can occur, and (2) a period during baseline to which one can compare behavior during extinction. Although Lerman and Iwata's definition appears better suited for a systematic investigation of the phenomenon then the aforementioned general definitions, it still fails to accurately outline either controlling variables or specific characteristics of the burst. 
Such shortcomings of this and other operational definitions are addressed in the following section.

\section{Problems with Existing Response Burst Definitions}

Although the above definitions accurately outline the general pattern of the extinction burst, questions remain concerning: (1) how large the increase in responding during the onset of extinction must be for it to be classified as a burst (2) whether dimensions of the target response aside from its frequency might be considered and (3) where such a burst can occur in relation to extinction onset to be counted as such.

For the first point above, most of the definitions described in the previous section state that the extinction burst is characterized by an increase in response rate; however, the magnitude and duration of this increase are not described. Existing definitions of the extinction burst fail to empirically specify important dimensions of the phenomenon. According to these definitions, any increase in responding compared to baseline, no matter how small or brief, can be categorized as an extinction burst. Such definitions of the burst are not sufficiently precise for a systematic understanding of the phenomenon.

Furthermore, all definitions aside from that proposed by Millenson (1967) focus on the extinction burst as an increase in response rate. This definition of the extinction burst does not consider other dimensions of responding such as duration, amplitude, or variability (Antonitis, 1951; Eckerman \& Lanson, 1969; Holton, 1961; Margulies, 1961; Mechner, 1958; Notterman \& Mintz, 1965; Stokes, 1995). Although the focus on bursting in terms of response rates is most common, this measure does overlook these other potential measures of the phenomenon.

The definition provided by Keller and Schoenfeld (1950) suffers from similar complications They suggest that extinction bursts occur because, "the animal is apt to attack 
vigorously the now-unrewarding bar" (Keller \& Schoenfeld, 1950, p. 71), thus proposing that the extinction burst is an aggressive response. Although, as noted above, aggressive behavior is a common generative effect of extinction (Azrin et al., 1966; Thompson \& Bloom, 1956), it seems overly restrictive to assert that all instances of bursting must include, or be, some form of aggressive behavior. It may make sense to characterize increases in response frequency or amplitude as manifestations of aggression, but increases in variations of response topography do not seem related to aggression. In the aforementioned experiment by Antonitis (1951), a burst may be said to have occurred due to an increase in the number of different spatial locations poked by the rat. Response amplitude was not recorded. As the presence of an aggressive response cannot be confirmed, this should not qualify as an example of an extinction burst according to Keller and Schoenfeld's definition. Regardless, it is cited as an example of bursts in response variability (e.g. Lerman \& Iwata, 1966).

There are many variables and measures to be considered when defining an extinction burst. These include the duration of each extinction session, the number of extinction sessions, and when in time extinction sessions occur after the completion of baseline. Extinction has been implemented differently across various experiments, in that some investigators have conducted multiple short sessions across several days (e.g. Anger \& Anger, 1976), whereas others have conducted a single long extinction session (e.g. Nevin, 1974). Therefore, it is important to consider the effect that different session lengths may have on measuring the extinction burst. Assume an extinction burst occurs in the first minute of an extinction session, after which the response rate gradually decays and eventually reaches zero at the beginning of the fifth minute. If the extinction session was only five minutes in length, the overall response rate for the session would be heavily influenced by the burst of responding that occurred within the first minute. 
Conversely, if the extinction session lasted for another ten minutes after the final response, the burst of responses would not exert as large of an influence on response rate if during the last ten minutes there was no responding. Response rates would be significantly higher in the former case than in the latter. These differences in response rate may cause only one case to meet the qualifications of an extinction burst when compared to baseline. Thus, differences in extinction session length may influence whether an extinction burst is observed.

Furthermore, if it assumed that the burst is a transient phenomenon that only occurs early in extinction (e.g. Cooper et al., 2007; Domjan \& Burkhard, 1982; Reynolds, 1968), it follows that the burst is localized to the initial portions of these early sessions. Thus, as opposed to characterizing the burst in terms of an increase in the response rate over an entire session, it may be more appropriate to define it in terms of rate of responding during only the first portion of the extinction session. Despite this, no experimenters have defined the burst in this manner, opting instead to focus on entire session response rates. As was described above, this focus on overall session response rates may mask the presence of an extinction burst in the case of lengthy extinction sessions by decreasing the overall response rate of the session.

A related question is one of the latency to the appearance of the extinction burst and its duration. On the one hand, Cooper et al. (2007) defined an extinction burst as, "an immediate increase in the frequency of the response after the removal of the positive, negative, or automatic reinforcement" (p. 462). Although this gives a general temporal frame for the extinction burst, "immediate" is not precise enough for a systematic evaluation of the phenomenon. On the other hand, the three-session period allotted for the extinction burst by Lerman and Iwata (1995), depending on what constitutes a session as was outlined above, could be too wide or too narrow a window for an increase in response rate to qualify as a burst. Take, for example, an instance in 
which response rate declines during the first session of extinction but then increases during the following session. According to Lerman and Iwata, this would qualify as an extinction burst. Conversely, it might be labeled as an instance of spontaneous recovery, rather than an extinction burst. Allowing for three whole sessions in which an increase in responding qualifies as an extinction burst complicates the study of the phenomenon.

Similarly, because the extinction burst is evaluated in terms of an increase in response rate compared to baseline, the number of sessions, or portions of the last session, in baseline to which a burst is compared must be defined concretely. Aside from the definition put forth by Lerman and Iwata (1995), existing definitions of the extinction burst fail to specify the baseline period to which the extinction burst should be compared. This lack of specificity is problematic as the number of baseline sessions, or the portions of baseline sessions, included in evaluating the extinction burst may influence reports of the phenomenon. Because response rates differ across baseline sessions, an assessment of bursting made by comparing response rates in the first session of extinction to only the final session of baseline may be different from one made to the average response rate of the final five sessions. Exclusively examining only the final session of baseline may not accurately reflect changes in response rate compared to baseline as a whole, and thus may influence interpretations of whether or not there has been an extinction burst. As stated above, only Lerman and Iwata specify the number of baseline sessions to which response rates in extinction should be compared. Although this is an improvement over other definitions, no systematic investigation of different numbers of baseline sessions has been conducted to determine if the number of sessions they allot is appropriate. Thus, existing definitions are not specific enough for a concrete understanding of the burst. 
Finally, how soon should extinction sessions begin after the completion of baseline? Following the final baseline session, extinction sessions may begin either immediately or following some interval of time (e.g., at the beginning of the next scheduled session). Differences in the time between the final session of baseline and the first session of extinction may contribute to differences in the discriminability of the change from baseline to extinction. The fact that most experiments that have reported bursts have used continuous reinforcement prior to the onset of extinction (e.g. Goh \& Iwata, 1994; Holton, 1961; Margulies, 1961, Zarcone et al., 1993), suggests that the discriminability of the beginning of extinction is a large contributor to the occurrence of a burst. The change from conditioning to extinction also might be more discriminable if there isn't a several-hours-long intervening period between the two conditions. This could be accomplished by conducting within-session transitions to extinction, in which responses are reinforced for the first portion of a session and then extinguishing for the remainder. As with differences in extinction-session duration, differences in the transition from baseline to extinction may also influence the extinction burst.

In sum, there are a number of questions and specifications to consider concerning the extinction burst. The magnitude of the increase; the dimension of responding to be examined; and the length, number, and time following the completion of baseline of each extinction session to be examined must all be specified to accurately define the extinction burst. These questions can be generalized into one, overarching question: What is an extinction burst? In other words, how should the phenomenon be operationally defined? Current definitions fail to adequately establish parameters for these aspects of the burst, and often contradict one another. Moving forward, such aspects will need to be concretely specified before any systematic investigation of the extinction burst can take place. For the purposes of the present experiment, an initial 
working definition of the extinction burst will follow the lead of some of the previous

definitions. Initially, it will be defined as an increase in response rate greater than or equal to the mean response rate across an equivalent portion of one or more sessions of baseline.

Furthermore, to qualify as a burst, this increase must occur within a period of time immediately following the removal of the reinforcer (no longer than the first session of extinction, but potentially shorter). This general definition is merely a starting point, however, because the purpose of the proposed experiments is to examine not only some of the conditions that might give rise to extinction bursts, but also to explore the utility of different definitions of the response burst.

\section{Prevalence of the Extinction Burst}

Although the extinction burst often is cited as one of the most frequently reported generative effects of extinction (Ducharme \& Van Houten, 1994; Kazdin, 1994; Martin \& Pear, 1992), in reality it does not appear to be as universal as this. A number of investigators have observed (Goh \& Iwata, 1994; Mace et al., 2010; Sajwaj, Twardosz, \& Burke, 1972; Zarcone et al., 1993) and failed to observe (Edwards et al., 1970; Mace et al., 2010; Rescorla \& Skucy, 1969) an extinction burst, however idiosyncratically defined, raising questions concerning the situations in which it may be expected to occur. Furthermore, in those experiments in which the phenomenon has been documented, criteria for observing the burst are similar to the general definitions outlined above (e.g. Zarcone et al., 1993), or are simply absent (e.g. Goh \& Iwata, 1994; Mace et al., 2010; Sajwaj, Twardosz, \& Burke, 1972). Mace et al. (2010), for example, exposed children with developmental disabilities who also were exhibiting various types of problem behavior to two extinction procedures, one following a baseline phase and the other following differential reinforcement of alternative behavior (DRA). Although the authors 
reported that extinction bursts occurred with the onset of extinction in roughly $50 \%$ of cases, a precise definition of the extinction burst was never specified. Thus, it is impossible to draw definitive conclusions concerning the prevalence of the phenomenon.

In the most comprehensive analyses of schedules of reinforcement (Ferster \& Skinner, 1975), which often included transitions from reinforcement to extinction, bursts are not mentioned. In describing an extinction curve for a pigeon previously exposed to an FR 40 schedule, Ferster and Skinner noted that "the usual terminal rate [of the FR 40] continues for only a few hundred responses before negative acceleration sets in" (p. 58). This continuation of "the usual terminal rate" suggests that a burst did not occur on introduction of extinction. In the numerous other examples of extinction following various FR schedules discussed throughout the chapter, Ferster and Skinner made similar statements concerning the progression of extinction. Furthermore, in the discussion of extinction following VR and variable-interval (VI) schedules, Ferster and Skinner (1957) again made no mention of an extinction burst. The chapter on VR schedules includes an extinction curve for a pigeon previously exposed to a VR 173 schedule. They stated that, "the record begins with a small portion of the VR 173 performance. This performance is followed by about 5000 responses at the original variable-ratio rate" (p. 413). For extinction following exposure to a VI 7-min schedule, the authors noted that, "during the first part of extinction the rate oscillates in a manner similar to that under the previous variableinterval reinforcement" (Ferster and Skinner, 1957, p. 347), but there was no mention of an extinction burst.

If the extinction burst is so prevalent, one would expect Ferster and Skinner (1957) to have reported it. Perhaps they did not mention the extinction burst because their focus was on the long-term overall course of extinction over several hours and hundreds of responses. Given 
such a broad scope, they may have overlooked or ignored any relatively brief changes in response frequency localized to the very beginning of the extinction condition. Furthermore, Ferster and Skinner's data on response rates are recorded in cumulative records. As the only way to analyze changes in response rate on cumulative records is by examining changes in slope - which is difficult to do precisely on such large time frames - the presence of an extinction burst may have been overlooked. This is especially likely if the magnitude of the bursts was not much greater than baseline levels of responding.

Another explanation as to why Ferster and Skinner (1957) did not report occurrences of the extinction burst concerns the schedules they investigated prior to extinction. Most experiments that have reported bursts have used an FR 1 as the schedule of reinforcement prior to the onset of extinction (e.g. Goh \& Iwata, 1994; Holton, 1961; Margulies, 1961, Zarcone et al., 1993), and the prevalence of bursting following other schedules has not been systematically investigated. Many of Ferster and Skinner's (1957) extinction conditions followed baseline schedules with ratio requirements much larger than 1 (e.g. FR 40 and VR 173). Thus, Ferster and Skinner did not investigate extinction following the schedule of reinforcement that most frequently produces an extinction burst.

To quantify data on the frequency of the extinction burst, Lerman and Iwata (1995) conducted meta-analyses on 113 applied studies employing extinction as an intervention. Using their definition as outlined above, Lerman and Iwata reported that of the 113 studies surveyed, only $27(24 \%)$ met the criterion for an extinction burst. In a second meta-analysis, bursts following extinction of self-injurious behavior (SIB) were examined in 41 data sets, where the burst was defined as in the Lerman and Iwata (1995) analysis (Lerman, Iwata, \& Wallace, 1999). Sixteen (39\%) of the data sets met the criterion for bursting. As both meta-analyses 
demonstrated that the extinction burst occurred in less than $50 \%$ of the surveyed data sets, the extinction burst may be a much less common phenomenon than prior findings have suggested.

It is possible that the unstandardized definitions of the extinction burst described above account for reports of a burst in some instances and not others. This possibility has not been investigated, as the conditions giving rise to the extinction burst have not been systematically examined. In fact, there are virtually no systematic experimental investigations of the extinction burst. Thus, the controlling variables that govern the extinction burst have not been identified.

\section{Controlling Variables of the Extinction Burst}

Relatively little is known about the variables that control a response burst at the onset of extinction. As was discussed in the Problems with Existing Definitions section, both the duration of each extinction session and the discriminability of the transition from baseline to extinction may influence the extinction burst. Other procedural variables, such as the schedule of reinforcement prior to the onset of extinction (continuous or intermittent, variable or fixed, interval or ratio, rich or lean, etc.), characteristics of the reinforcer that previously maintained the behavior (frequency, duration, and delay to reinforcement) and the method of implementing extinction (removal of the positive reinforcer versus elimination of the response-reinforcer dependency), all may influence the discriminability of extinction and thus the occurrence of an extinction burst.

Although these variables may control the occurrence of the extinction burst, no experiments have evaluated this possibility. The only studies to investigate controlling variables of the burst are the two previously described meta-analyses. Both suggest that an extinction burst was less likely when extinction was introduced at the same time as another form of treatment (e.g. differential reinforcement of other behavior [DRO], DRA, response-independent 
reinforcement) (Lerman \& Iwata, 1995; Lerman et al., 1999). Additionally, Lerman et al. (1999) postulated that the likelihood of a burst was influenced by the conditions in effect prior to the onset of extinction. They noted that bursts were more frequent "for cases in which SIB was maintained by social negative reinforcement (12 of 21 cases, or 57\%) than for cases in which SIB was maintained by social positive reinforcement (4 of 17, or 23\%)" (Lerman et al., 1999, p. 5).

Definitive conclusions about the role of these potential controlling variables in the mediation of an extinction burst are precluded by the methods of both meta-analyses. Firstly, the definition of the extinction burst used in both meta-analyses suffers from the shortcomings described in the section on definitions above. Secondly, Lerman and Iwata (1995) reported that, "if at least one of the data sets for a given subject met the burst criterion, a single occurrence of the extinction burst was scored for the case" (p. 93). Given that Mace et al. (2010) demonstrated the intermittence of the extinction burst within individual participants, Lerman and Iwata may have overestimated the frequency of bursts in their sample. Thirdly, the authors do not include any supplementary details on experiment uniformity in their meta-analyses. Thus, any important procedural differences between experiments that might mitigate the extinction burst are lost in the amalgamation of the data from a variety of procedures, populations, and treatments that were not separated in the meta-analyses.

As the literature reviewed in the preceding sections illustrates, relatively little is known about the extinction burst. Given the current understanding of the phenomenon, it is not possible to predict the dimensions of the operant (e.g., Gilbert, 1958) that are likely to reveal a burst in response rate, the baseline reinforcement conditions that make a burst more or less likely, the magnitude of a burst, and if the intensity of a burst can be attenuated. Thus, the purpose of the 
following experiments is to gain a better understanding of the phenomenon. As described above, Anger and Anger (1976) developed a procedure to study the effect of repeated blocks of extinction on behavior and found that numbers of responses were relatively consistent across blocks. Because this procedure showed that behavior persisted and did not completely extinguish after as many as eight blocks of extinction, it can be adapted to investigate whether an extinction burst can be reliably reproduced across successive rounds of extinction. Therefore, the present experiment will adapt this procedure as a starting point to investigate the reproducibility of the extinction burst and identify a set of circumstances under which it can be consistently replicated.

\section{Statement of the Problem}

Extinction is the reduction or elimination of responding when a previously reinforced response no longer is reinforced. If extinction is defined by eliminating the reinforcer entirely, response rates quickly approach zero, but if it is defined by eliminating only the responsereinforcer dependency, response rates decline more gradually (Lattal, 1972). Other properties of the extinction procedure that may influence how behavior changes during extinction include the reinforcement conditions in effect prior to extinction, whether extinction is introduced at the same time as an alternative contingency (Lerman \& Iwata, 1995; Lerman et al., 1999), whether extinction is introduced gradually or abruptly (Terrace, 1963), and the number of successive times the extinction procedure is implemented (Anger \& Anger, 1976).

The effects of extinction are not limited to diminished rates of responding. During extinction procedures, certain responses - be they the target or some other alternative sometimes increase in frequency. These phenomena are collectively termed the generative effects of extinction and often are contrasted with the eliminative effects described above. Such 
generative effects of extinction include increases in the variability of the topography of the target response (Antonitis, 1951; Eckerman \& Lanson, 1969), the emergence of alternative responses (Azrin, et al., 1966; Goh \& Iwata, 1994; Thompson \& Bloom, 1966), and the extinction burst (Cooper et al., 2007; Keller \& Schoenfeld, 1950).

The extinction burst has been considered an example of such a generative effect of extinction and may be broadly defined as an immediate increase in response rate following the onset of extinction (Cooper et al., 2007; Lattal, St Peter, \& Escobar, 2013). Although the extinction burst most frequently is referenced as an increase in response rate, it also has been manifested as an increase in response duration, amplitude, or variability (Antonitis, 1951; Eckerman \& Lanson, 1969; Holton, 1961; Margulies, 1961; Mechner, 1958; Notterman \& Mintz, 1965; Stokes, 1995). Although the extinction burst has been described as the most frequently demonstrated and reliable generative effect of extinction (Ducharme \& Van Houten, 1994; Kazdin, 1994; Martin \& Pear, 1992), the experimental evidence for such a statement is mixed, at best. Different experimenters have observed (e.g., Goh \& Iwata, 1994; Mace et al., 2010; Sajwaj et al., 1972; Zarcone et al., 1993) and failed to observe (e.g., Edwards et al., 1970; Mace et al., 2010; Rescorla \& Skucy, 1969) the extinction burst, raising questions concerning its reliability and the circumstances under which it occurs. Despite these mixed findings, there has been almost no attention directed toward identifying the controlling variables of the extinction burst.

In two meta-analyses, Lerman and Iwata (1995) and Lerman et al. (1999) examined the frequency of extinction bursts in studies employing extinction as a form of treatment. Between 24\% (Lerman \& Iwata, 1995) and 39\% (Lerman et al., 1999) of the studies surveyed in both meta-analyses reported an extinction burst, which these authors defined as "an increase in responding during any of the first three treatment sessions above that observed during all of the 
last five baseline sessions (or all of baseline if it was briefer than five sessions)" (Lerman \& Iwata, 1995, p. 93). Thus, both meta-analyses showed that the extinction burst is a much less reliable phenomenon than prior observers have suggested.

Definitive conclusions about the prevalence and controlling variables of the extinction burst are precluded by limitations of the meta-analyses described in the preceding paragraph. The operational definitions used in both analyses to identify if an extinction burst occurred are too broad and imprecise to accurately capture the phenomenon. Furthermore, important procedural differences between the experiments included in the meta-analyses prevent conclusions from being drawn concerning the controlling variables. Variables such as baseline and extinction session duration and number, when in time extinction sessions followed baseline, the schedule of reinforcement prior to the onset of extinction (variable or fixed, interval or ratio, rich or lean, etc.), the method of implementing extinction (removal of the positive reinforcer versus elimination of the response-reinforcer dependency), and the stability of responding at the termination of baseline all are candidates for determining characteristics of the burst, but all were intermingled among the experiments that Lerman and Iwata reviewed. All or none of these may have modulated the occurrence of an extinction burst.

There is little basic research under controlled conditions concerning the nature of the extinction burst. A better understanding of the phenomenon requires that controlling variables modulating its occurrence be identified. Thus, the purpose of the current experiments was to begin the task of isolating such variables. A starting point was to determine whether the extinction burst can be produced reliably and replicated across repeated exposures to extinction. This was accomplished by adapting a procedure described by Anger and Anger (1976) for studying the effects of repeated blocks of extinction on behavior. In each of the following 
experiments, blocks of extinction were alternated with conditioning phases in which responding was maintained by a ratio schedule of reinforcement. This repeated-extinctions procedure allowed for the examination of extinction bursts across successive exposures to extinction. Furthermore, the reinforcement schedule in effect during baseline sessions was different in each of three experiments to investigate how parameters of the extinction burst changed as a function of training conditions.

\section{General Method}

\section{Subjects}

Six experimentally naïve male White Carneau pigeons were maintained at $80 \%$ of freefeeding weight. Each was housed in separate cages in a vivarium under a 12:12-hr light/dark cycle and had continuous access to water and health grit in their home cages. Three pigeons were used in each experiment (see Table 1 for details).

\section{Apparatus}

Two plywood operant conditioning chambers, each enclosed in a separate soundattenuating, ventilated enclosure were used. Chamber 1 was $30.8 \mathrm{~cm}$ wide by $32.4 \mathrm{X} \mathrm{cm}$ long by $38.1 \mathrm{~cm}$ high, and Chamber 2 was $31.1 \mathrm{~cm}$ wide by $32.4 \mathrm{~cm}$ long by $37.5 \mathrm{~cm}$ high. The aluminum work panels of each chamber contained either 3 (Chamber 1) or 2 (Chamber 2) response keys, each $1.9 \mathrm{~cm}$ in diameter. The keys were mounted $8.9 \mathrm{~cm}$ apart horizontally in Chamber 1. The keys in Chamber 2 each were mounted $5.1 \mathrm{~cm}$ to the left and right of the center of the panel. Each key required a force of approximately $0.15 \mathrm{~N}$ to operate and was transilluminated white. Only one key (the center in Chamber 1 and the left in Chamber 2) was used in the experiment. Reinforcement was 3-s access to Purina Nutri-Blend ${ }^{\mathrm{TM}}$ pellets delivered from a Gerbrands model G5610 food hopper accessible through either a 5.7 by 4.4-cm (Chamber 
Table 1

Pigeons used in each of the three experiments.

\begin{tabular}{ccccccc} 
& \multicolumn{7}{c}{ Pigeon } \\
\cline { 2 - 7 } Experiment & 10197 & 10028 & 13715 & 20542 & 18390 & 1576 \\
\hline 1 & Yes & Yes & Yes & - & - & - \\
2 & Yes & Yes & Yes & - & - & - \\
3 & - & - & - & Yes & Yes & Yes \\
\hline
\end{tabular}


1) or 5 by $5-\mathrm{cm}$ (Chamber 2$)$ aperture. The center of the aperture was located either $15.9 \mathrm{~cm}$ from the left edge of the work panel and $9.8 \mathrm{~cm}$ from the floor of the chamber (Chamber 1), or $16.5 \mathrm{~cm}$ from the left edge of the work panel and $10.8 \mathrm{~cm}$ from the floor of the chamber (Chamber 2). A houselight was on throughout each session except during food presentations. The center of the houselight was located either $27.3 \mathrm{~cm}$ from the left edge of the work panel and $4.4 \mathrm{~cm}$ from the floor of the chamber (Chamber 1) or $26.7 \mathrm{~cm}$ from the left edge of the work panel and $5.7 \mathrm{~cm}$ from the floor of the chamber (Chamber 2). A ventilation fan and white noise generator masked extraneous noise. Contingencies were programmed, and data recorded, on a desktop computer operating with Med-PC@ software.

\section{Procedure}

Sessions occurred seven days a week at approximately the same time each day. Each session started with a 3-min blackout in the chamber to minimize effects of handling on early responding. The start of each session was signaled by the onset of the houselight and, in sessions within all phases after Conditioning Phase 1, the keylight.

Magazine training and shaping. Magazine training continued for each pigeon until it reliably approached and ate from the hopper within $1 \mathrm{~s}$ of it being raised. Following magazine training, key pecking responses were shaped manually via the reinforcement of successive approximations. During shaping, the key was transilluminated white. Shaping sessions continued until keypecking and eating from the hopper occurred within $1 \mathrm{~s}$ of keylight transillumination and hopper presentation for the duration of the session.

Conditioning Phase 1. After keypecking was shaped, each pigeon was exposed to an autoshaping procedure in one of the operant chambers. All subsequent sessions occurred in these chambers, with chamber held constant for each pigeon throughout the experiments. The 
autoshaping procedure was a discrete trials procedure in which intertrial intervals averaged $30 \mathrm{~s}$ and were drawn from the distribution described by Fleshler and Hoffman (1962). Pecks before the variable-time (VT) interval elapsed had no consequence. Once the VT elapsed, one of the response keys was transilluminated white for $10 \mathrm{~s}$. If a peck was made to the white key, the light turned off and the hopper was raised for $3 \mathrm{~s}$. If no peck was made to that key, it turned off and the hopper was raised at the end of the 10-s interval. After the hopper was deactivated, a new VT interval began. The autoshaping procedure was completed after the $10^{\text {th }}$ consecutive trial on which a peck occurred. If on any trial the pigeon did not peck the key, the autoshaping procedure was restarted and pecks on the next 10 consecutive trials were required to complete the procedure.

After completing the autoshaping procedure, each pigeon was, in the same session, moved on to an FR training procedure in which, in the presence of a white keylight, each peck to the response key produced 3-s access to the food hopper (an FR 1 schedule). Once the pigeon earned 10 reinforcers, the FR training procedure was terminated, marking the end of Conditioning Phase 1.

Conditioning Phase 2. The following day after completing Conditioning Phase 1, a ratio schedule of reinforcement was put in place. Aspects of the specific schedule enacted varied from experiment to experiment and thus will be detailed in the respective sections of this document. Across all experiments, ratio requirements were constructed based on the distribution described by Fleshler and Hoffman (1962). Sessions lasted until 60 reinforcers were earned or until $1 \mathrm{hr}$ elapsed, whichever came first, and were conducted for five consecutive days before progressing to the next condition. In the case of technical problems or data that were aberrant from that of 
other sessions, the phase was extended by at most three sessions. During some blocks, due to technical problems, only four sessions were conducted.

Extinction phase. After the fifth session of Conditioning Phase 2, keypecking was extinguished over at least eight sessions by eliminating the delivery of reinforcement for keypecking. The length of these sessions varied from experiment to experiment and will be detailed below.

After the eighth Extinction phase session, this three-phase sequence was repeated as described above. Each pigeon progressed through these three phases in this order up to 9 times, with a minimum of at least 5 exposures to the Extinction phase.

Data analysis. The occurrence and magnitude of response bursts was evaluated by comparing response rates between equivalent portions of Conditioning Phase 2 and each session during each Extinction phase. In addition to overall response rates during a session, analyses of response rates were conducted for the first 10,30,60, and $300 \mathrm{~s}$ of both phases; however, of the latter only the analyses for the first $60 \mathrm{~s}$ of both phases are included in this document. Extinction bursts were identified by comparing response rates during solely the first session of the Extinction phase to response rates during the preceding block of Conditioning Phase 2. First, potential bursts were screened via comparisons to both the response rate of the final session of the preceding block of Conditioning Phase 2 and the mean response rate across all five sessions of the phase. If rates during the first session of extinction were at least $5 \%$ higher than either of these measures, then an additional comparison to the maximum response rate of Conditioning Phase 2 was also conducted. Response rates during the first session of extinction that were at least $5 \%$ higher than the maximum response rate measured in the previous reinforcement period were classified as extinction bursts. 
Additionally, because the experimental design employed repeated exposures to extinction, specific attention was given to changes in bursting or response rates (a) across successive blocks of extinction, (b) across successive sessions of extinction within a single block, and (c) over the course of a single session.

\section{Experiment 1}

In Experiment 1, blocks of extinction alternated with baseline conditioning phases in which responding was maintained by an escalating VR schedule of reinforcement to investigate both the time course of repeated extinctions and the reproducibility of the extinction burst.

\section{Procedure}

Conditioning Phase 1. The combined autoshaping and FR sessions were conducted as described in the General Method section.

Conditioning Phase 2. At the beginning of each session a VR 5 schedule was in effect. The keylight was transilluminated white and an average of five pecks resulted in 3-s access to food. This VR 5 schedule remained in effect until 20 reinforcers were earned, after which it was changed to VR 10. After 20 reinforcers were earned on the VR 10 schedule, the schedule was changed to VR 20. The session terminated following the $20^{\text {th }}$ reinforcer on the VR 20 schedule. This sequence was repeated on each day of Conditioning Phase 2 .

Extinction phase. Sessions in the Extinction phase were conducted as described in the General Method section, with each session lasting as long as the mean session duration during Conditioning Phase $2(M=8.20 \mathrm{~min}$, minimum $=4.89$, maximum $=15.15 \mathrm{~min})$. The first session in the Extinction phase was conducted the day following the final session of Conditioning Phase 2. 
After the eighth extinction session, Conditioning Phases 1 and 2 were repeated, exactly as described above. After these six consecutive sessions, the Extinction phase was repeated. Each pigeon progressed through these three phases either eight (10028 and 13715) or nine times (10197).

\section{Results}

Time-course of extinction. Changes in response rates were assessed across successive blocks of extinction, across successive sessions of extinction within a single block, and over the course of a single extinction session. Comparisons across successive blocks and sessions were conducted for both responses throughout the entire session (whole-session level of analysis) and solely within the first minute of the session (first-minute level of analysis). Data in support of these comparisons are found in Figures 1 and 2.

Changes across successive blocks. The conventional negatively-decelerating extinction curve (see Cooper et al., 2007) was replicated across successive repeated extinctions for all three pigeons. At both the whole-session and first-minute level, the curve became less pronounced following repeated exposures to extinction for two pigeons (10028 and 13715). This effect was driven predominantly by decreases in the response rate for the first session of each block of the Extinction phase. For the final pigeon (10197) the extinction curve maintained the same general form across the repeated exposures. Overall there was more variability in response rate at the first-minute level of analysis compared to the whole-session level.

Changes across successive sessions within a block. At both levels of analysis, response rates declined across successive sessions within single 8-session blocks of extinction for all three pigeons. These declines in response rates followed a similarly negatively-accelerated function as described for the changes across successive blocks of extinction. 


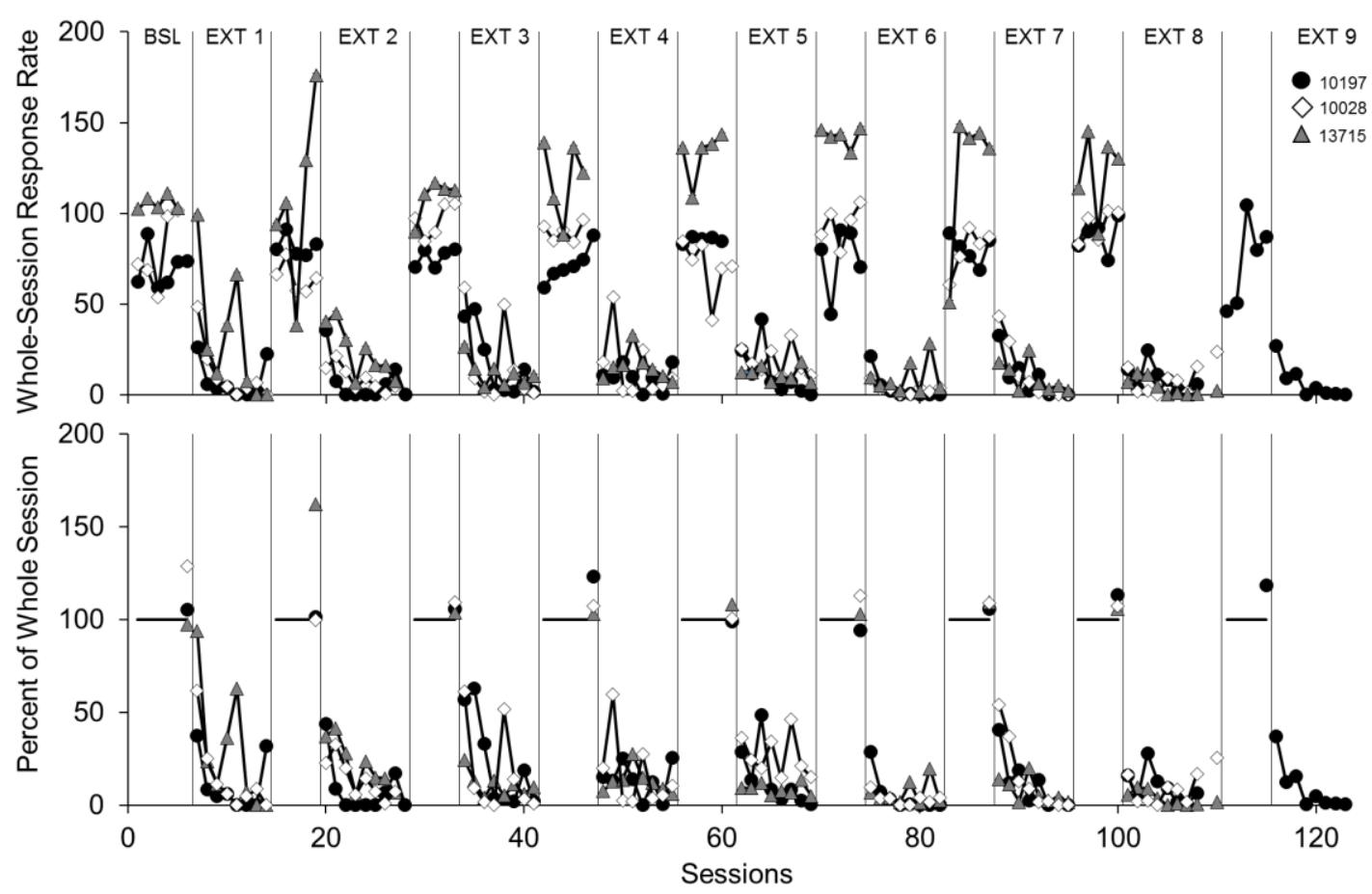

Figure 1. Response rates for each pigeon across blocks of the repeated-extinctions procedure at the whole-session level of analysis. The upper panel shows absolute response rates per minute for the sessions shown. The lower panel shows the upper-panel data transformed to a percentage of the mean response rate of each block of Conditioning Phase 2. Solid horizontal lines in Conditioning Phase 2 sections of the lower panel show the mean baseline response rate. Data points superimposed over this line are the response rates for the final session of Conditioning Phase 2 calculated as a percentage of the mean response rate for the phase. In those blocks containing fewer than five (Conditioning Phase 2) or eight (Extinction phase) data points, technical problems resulted in lost data for those sessions. Note that only the first baseline (BSL) is identified. All of the panels without labels are subsequent baseline conditions. 


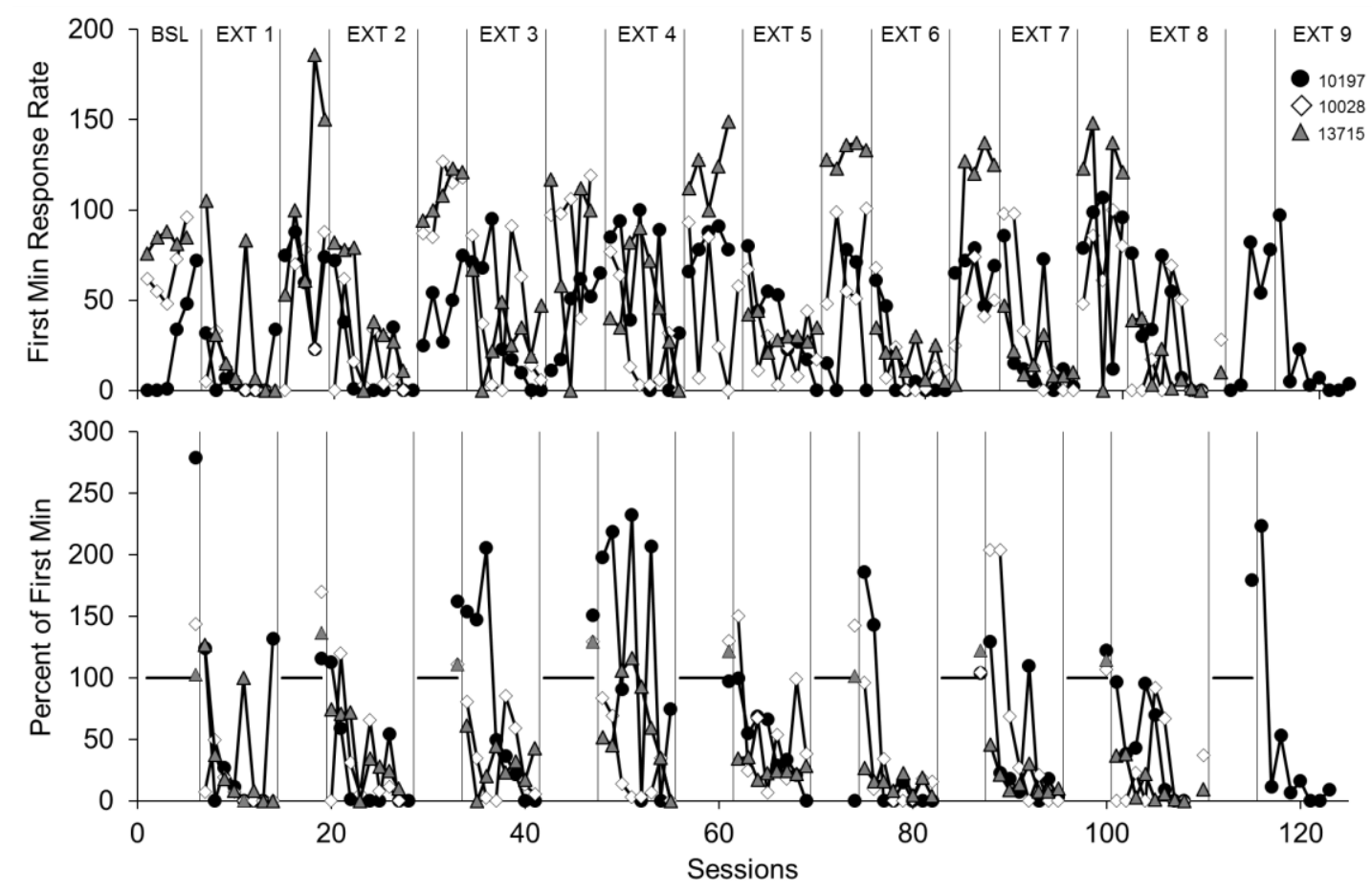

Figure 2. Response rates for each pigeon across blocks of the repeated-extinction procedure at the first-minute level of analysis. The upper panel shows the absolute response rate per minute for the sessions shown. The lower panel shows the upper-panel data transformed to a percentage of the mean response rate of each block of Conditioning Phase 2. Solid horizontal lines in Conditioning Phase 2 sections of the lower panel show the mean baseline response rate. Data points superimposed over this line are the response rates for the final session of Conditioning Phase 2 calculated as a percentage of the mean response rate for the phase. In those blocks containing fewer than five (Conditioning Phase 2) or eight (Extinction phase) data points, technical problems resulted in lost data for those sessions. Note that only the first baseline (BSL) is identified. All of the panels without labels are subsequent baseline conditions. 
Changes within a single session. Within individual sessions of the Extinction phase, minute-by-minute response rates declined over time for all three pigeons. Generally, response rates within the first minute of the Extinction phase were substantially higher than those late in the Extinction phase. As with changes in response rate across blocks of extinction and sessions within a block, changes within a single session also followed a similarly negatively-accelerated function.

Whole-session analysis of response rates during extinction. Response rates (responses in a session/session time in minutes) for each pigeon across all blocks of Conditioning Phase 2 and the subsequent Extinction phase are shown in the upper panel of Figure 1. The lower panel of the figure shows these same data transformed to a percentage of the mean response rate of each block of Conditioning Phase 2 [(response rate in extinction/mean Conditioning Phase 2 response rate) $\mathrm{x}$ 100] for ease of comparison across pigeons and blocks of extinction. In each Conditioning Phase 2 section of the lower panel of Figure 1, the solid line at 100 on the Y-axis represents the mean response rate of the sessions in this phase. The data points superimposed over this line reflect the response rates for the final session of Conditioning Phase 2 calculated as a percentage of the mean response rate for the phase.

For all three pigeons, an extinction burst did not occur during the first Extinction phase. Response rates during the first session of the Extinction phase were substantially less than mean Conditioning Phase 2 response rates for both 10197 and 10028 (37\% and 62\% of mean Conditioning Phase 2 response rates respectively) and were slightly less than mean Conditioning Phase 2 rates for 13715 (94\% of mean Conditioning Phase 2 response rates). Similar results were obtained for comparisons to maximum Conditioning Phase 2 response rates (30\%, 48\%, and $89 \%$ of maximum Conditioning Phase 2 response rates for 10197, 10028, and 13715 
respectively). Moreover, there were no extinction bursts during any subsequent exposures to the Extinction phase.

First-minute analysis of response rates during extinction. Figure 2 shows response rates across all blocks of the experiment calculated for the first minute (responses in the first minute of a session/one minute) of either Conditioning Phase 2 or the Extinction phase (depending on the session). As in Figure 1, the upper panel shows absolute response rates and the lower shows data transformed as a percentage of responding in each block of Conditioning Phase 2.

When analyses were restricted to responses during the first minute of both Conditioning Phase 2 and the Extinction phase, bursting occurred in all three pigeons. Comparisons of firstextinction-session response rates to the burst criteria are detailed in Table 2. As the data in the table show, the frequency, timing (i.e. the blocks of the Extinction phase in which bursts occurred), and magnitude of these bursts (hereafter referred to as the dimensions of bursting) were idiosyncratic across pigeons. Furthermore, the timing and magnitude of these bursts differed across successive extinction bursts within individual pigeons. As such, the extinction burst was an intermittent phenomenon that occurred only during certain exposures to extinction, with no systematic pattern to its occurrence.

\section{Discussion}

Successive alternations between blocks of an increasing VR schedule of conditioning and blocks of extinction intermittently produced extinction bursts in three pigeons. Such bursts occurred, however, only when the analysis was restricted to responses within the first minute of both the conditioning and extinction sessions. Furthermore, the occurrence, timing, and magnitude of the burst was inconsistent both within and between subjects. Each pigeon 


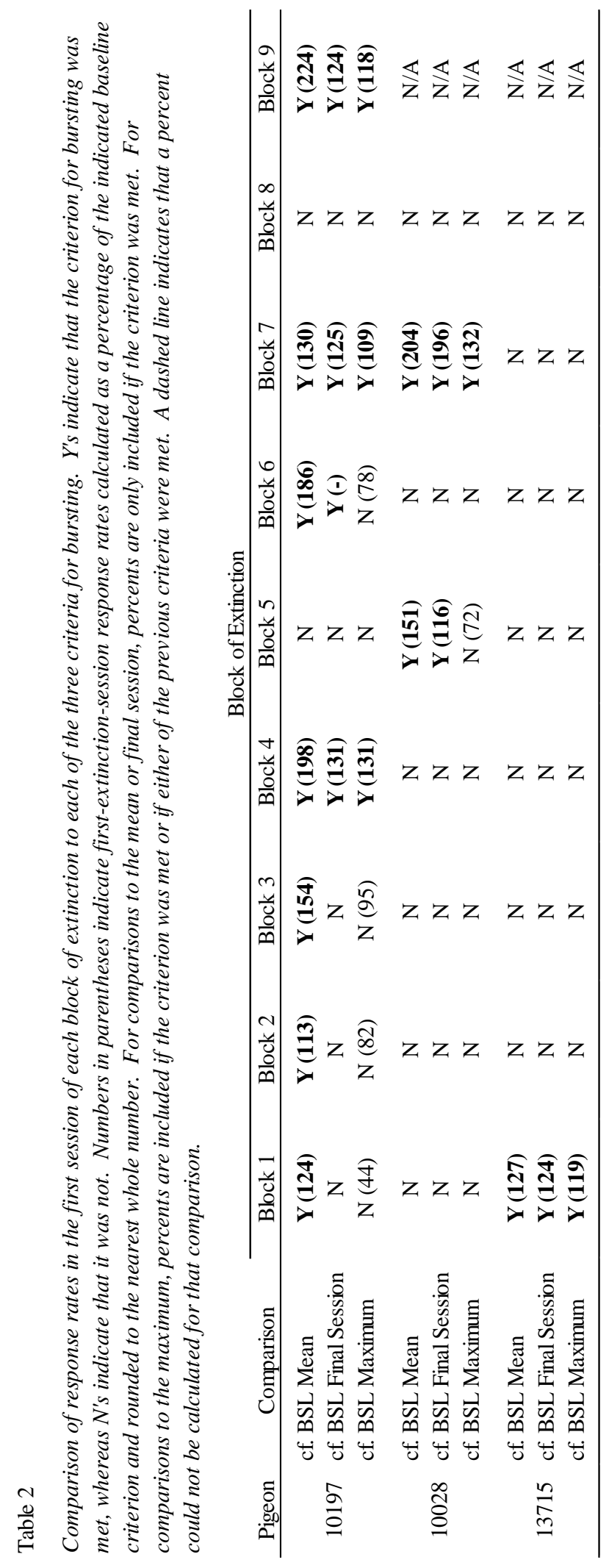


exhibited different frequencies, magnitudes, and times of occurrence of bursting throughout the repeated-extinctions procedure. Additionally, these dimensions of bursting were not constant in those pigeons exhibiting extinction burst during more than a single block of extinction.

Measurement considerations. Given the paucity of basic research on the extinction burst, a discussion of the methods used to assess and measure instances of the phenomenon is warranted. Points of interest in this discussion include a comparison of the whole-session and first-minute levels of analysis, an evaluation of the merits of the first-minute level of analysis, and an assessment of the criteria for bursting employed. Collectively, these points can be grouped together as considerations regarding the measurement of the extinction burst. Given their central relevance across all three experiments, these considerations are addressed in more detail in the General Discussion section.

Constraints on generality. Although the repeated-extinctions procedure did lead to instances of bursting across all three subjects, certain aspects of the procedure as it was employed currently have implications for the generality of the findings. First, given the use of a modified VR schedule during Conditioning Phase 2, it is difficult to pinpoint when in the Extinction phase the extinction procedure was contacted and the eliminative effects of extinction began. When extinction is implemented following an FR schedule, the extinction contingency is contacted for the first time immediately after the final response in the discontinued ratio requirement is made and a reinforcer is not delivered. As such, only those responses made after the completion of the first ratio requirement should be included in an analysis of bursting.

The same holds true concerning extinction following a VR schedule. Given the variable nature of reinforcer delivery during conditioning however, there is no fixed point of contact with the extinction contingency following conditioning on VR schedules. Although the average ratio 
requirement for the VR schedule may at first glance appear to be a promising point of contact with the contingency, reinforcers can be earned at higher response requirements during Conditioning Phase 2 sessions. As such, the absence of reinforcer delivery after the response that meets the average ratio requirement does not necessarily signal extinction. Alternatively, the maximum response requirement for the schedule may be said to function as the point of contact with the extinction contingency. However, given that most reinforcers are delivered following the completion of lower response requirements, this may not be the point of contact either. Although neither of these two response requirements precisely specify the point of contact with extinction, that contact point should be in some way related to the response requirements of the schedule in place during conditioning. As such, it is more difficult to determine which responses should be included in an analysis of bursting following VR schedules.

The modified VR schedule of reinforcement with its incrementing response requirement at the beginning of each session further complicates this issue, as the use of three separate VR schedules during each session of Conditioning Phase 2 further blurs the point of contact with the extinction contingency. Thus, before the point of contact can be determined, the correct schedule to guide the search for this point must be decided upon. Given that the VR 5 schedule is the first schedule in effect during each Conditioning Phase 2 session, it is possible that this is the schedule influencing when the extinction contingency is contacted. Alternatively, given that the VR 20 schedule is the final schedule in effect before the enactment of extinction, attention perhaps should be directed to this schedule instead.

The increasing VR schedule employed during Conditioning Phase 2 was adapted from the baseline used in the original experiment by Anger and Anger (1976). As their procedure 
previously had been shown to maintain responding during extinction even across repeated exposures to extinction, it was used in the current experiment in which responding needed to be maintained or at least re-established during subsequent Conditioning phase 2 exposures to study any instances of bursting. Despite the limitations of the procedure as it was implemented, responding was maintained over the course of up to nine blocks of extinction and increases in responding early during extinction that may be interpreted as extinction bursts occurred. Thus, the repeated-extinctions procedure does appear to be a promising method by which to investigate the extinction burst across repeated extinctions. The next two experiments addressed the limitations of the increasing VR 20 scheduled used in Conditioning Phase 2, and therefore further investigate the extinction burst across repeated exposures to extinction.

\section{Experiment 2}

To address some of the procedural limitations of Experiment 1, a conventional VR 20 schedule was used as the baseline reinforcement schedule in Conditioning Phase 2 for Experiment 2. Additionally, to investigate the effect of the shift to extinction on the occurrence of the extinction burst, two methods for transitioning from Conditioning Phase 2 to the Extinction phase (between-session and within-session) were used.

\section{Procedure}

Conditioning Phase 1. The combined autoshaping and FR sessions were conducted as described in the General Method section.

Conditioning Phase 2. This phase was conducted as described in the General Method section, but with a conventional VR 20 schedule in effect throughout each session. Unlike the schedule in place during Experiment 1, the ratio requirement did not change over the course of each session. 
Extinction. Keypecking was extinguished across eight sessions as described in the General Method section, with each session lasting for 20 min. After the eighth extinction session, this three-phase sequence was repeated at least five times (see Table 3 for the number of times each pigeon was exposed to the sequence).

Depending on the extinction block, the first session of the Extinction phase was conducted on either the day following the final session of Conditioning Phase 2, or on the same day immediately after the final session was completed. This former type, a "between-sessions transition to extinction," was conducted as described in Experiment 1. That is, the first extinction session began on the following day. For the latter type, a "within-session transition to extinction," the first extinction session began immediately following the delivery of the $60^{\text {th }}$ reinforcer of the final session of Conditioning Phase 2. Aside from the cessation of reinforcer delivery for pecking, the stimulus conditions remained unchanged from the immediately preceding conditioning session.

The sequence described in the preceding paragraph was only in place during the final session of Conditioning Phase 2 and the (immediately following) first session of the Extinction phase. All subsequent Extinction phase sessions within that block occurred on separate days as detailed in the General Method section. The sequence of Conditioning Phases $1 \& 2$ followed by extinction was repeated several times for each pigeon. Table 3 shows both the order of these between- and within-session transitions and the number of exposures to each for each pigeon.

\section{Results}

Time-course of extinction. For each pigeon, repeated exposures to extinction produced the same behavioral effects as observed in Experiment 1: response rates during Extinction phase sessions decreased across successive blocks, across successive sessions within a block, and over 
Table 3

Sequence, type, and number of transitions for each pigeon in Experiment 2

\begin{tabular}{ccccc} 
& \multicolumn{2}{c}{ First Transition } & \multicolumn{2}{c}{ Second Transition } \\
\cline { 2 - 5 } Pigeon & Type & Number & Type & Number \\
\hline 10197 & Within-Session & 8 & N/A & N/A \\
10028 & Between-Sessions & 5 & Within-Session & 6 \\
13715 & Between-Sessions & 6 & Within-Session & 7 \\
\hline
\end{tabular}


time within individual sessions (see Figures 3, 4, 5, and 6). One notable exception to this pattern was the response rate in the first two sessions of the fifth block of extinction for 13715 (see Figures 5 and 7). The increase in response rates for these two sessions compared to those of the previous block may be attributed to a one-month hiatus due to injury that transpired between the completion of the fourth block of extinction and the beginning of the fifth block of Conditioning Phase 2.

\section{Between-sessions transitions.}

Whole-session analysis of response rates during extinction. Absolute response rates for each pigeon across all blocks of between-sessions transitions to extinction are shown in the upper panel of Figure 3. Response rates in the lower panel were calculated as a percentage of the average response rate for each block of Conditioning Phase 2.

Extinction bursts did not occur during the first block of the Extinction phase for either pigeon. Response rates during the first session of the Extinction phase were substantially less than mean Conditioning Phase 2 response rates for both 10028 and 13715 (15\% and 37\% of mean Conditioning Phase 2 response rates respectively). Similar results were obtained for comparisons to maximum Conditioning Phase 2 response rates (12\% and 34\% of maximum Conditioning Phase 2 response rates for 10028 and 13715 respectively). Additionally, there were no extinction bursts across any subsequent exposures to extinction.

First-minute analysis of response rates during extinction. Figure 4 shows response rates for both pigeons across all between-sessions transitions to extinction, calculated for only the first minute of either Conditioning Phase 2 or the Extinction phase (depending on the session). The upper panel shows absolute response rates in Conditioning Phase 2 and Extinction phase sessions. Response rates in the lower panel were transformed to a percentage of response 


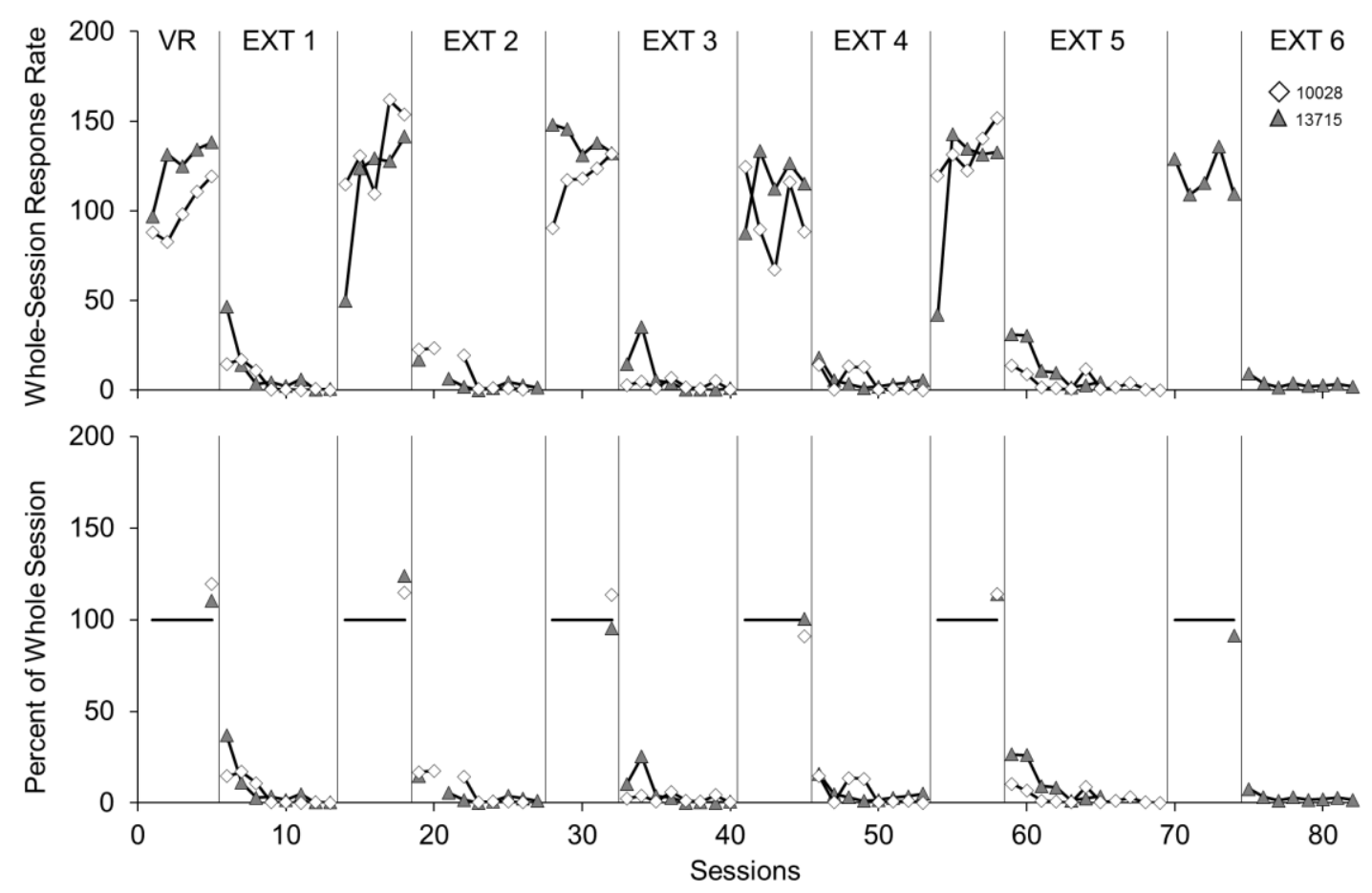

Figure 3. Response rates for both pigeons across all blocks of between-sessions transitions at the whole-session level of analysis. The upper panel shows the absolute response rate per minute for the sessions shown. The lower panel shows the upper-panel data transformed to a percentage of the mean response rate of each block of Conditioning Phase 2. Solid horizontal lines in Conditioning Phase 2 sections of the lower panel show the mean baseline response rate. Data points superimposed over this line are the response rates for the final session of Conditioning Phase 2 calculated as a percentage of the mean response rate for the phase. In those blocks containing fewer than five (Conditioning Phase 2) or eight (Extinction phase) data points, technical problems resulted in lost data for those sessions. Note that only the first baseline (VR) is identified. All of the panels without labels are subsequent baseline conditions. 

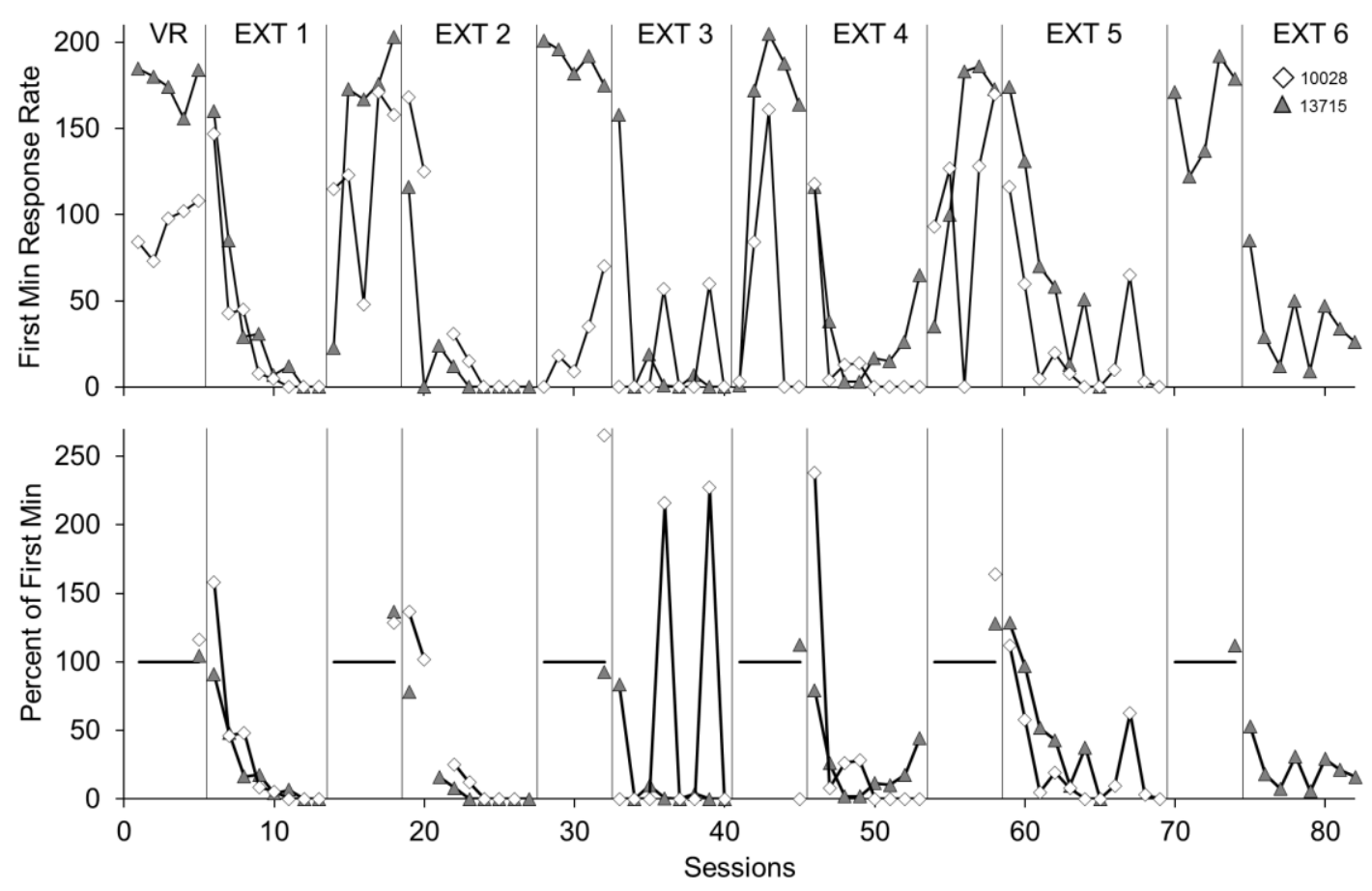

Figure 4. Response rates for both pigeons across all blocks of between-sessions transitions at the first-minute level of analysis. The upper panel shows the absolute response rate per minute for the sessions shown. The lower panel shows the upper-panel data transformed to a percentage of the mean response rate of each block of Conditioning Phase 2. Solid horizontal lines in Conditioning Phase 2 sections of the lower panel show the mean baseline response rate. Data points superimposed over this line are the response rates for the final session of Conditioning Phase 2 calculated as a percentage of the mean response rate for the phase. In those blocks containing fewer than five (Conditioning Phase 2) or eight (Extinction phase) data points, technical problems resulted in lost data for those sessions. Note that only the first baseline (VR) is identified. All of the panels without labels are subsequent baseline conditions. 


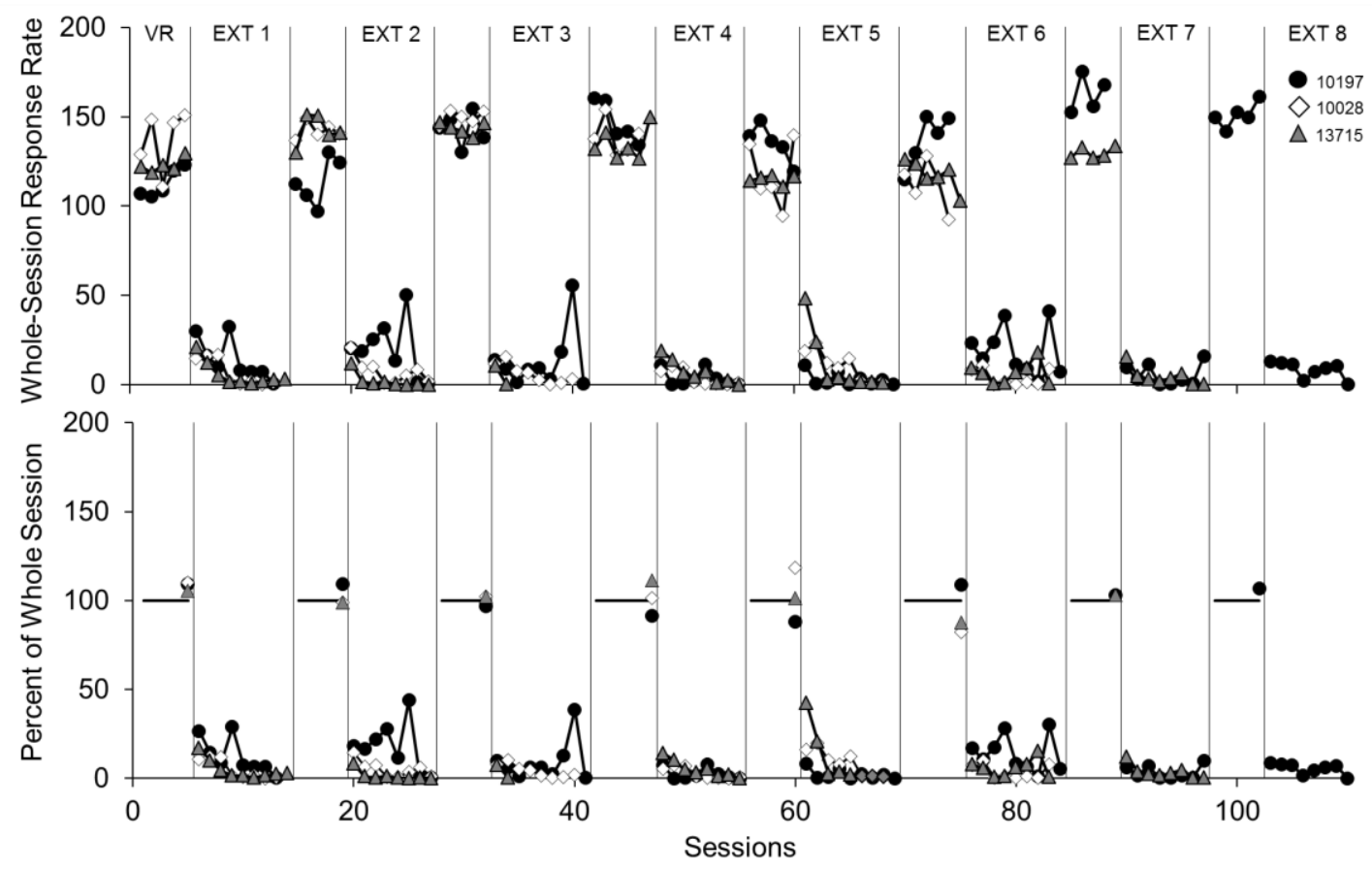

Figure 5. Response rates for each pigeon across all blocks of within-session transitions at the whole-session level of analysis. The upper panel shows the absolute response rate per minute for the sessions shown. The lower panel shows the upper-panel data transformed to a percentage of the mean response rate of each block of Conditioning Phase 2. Solid horizontal lines in Conditioning Phase 2 sections of the lower panel show the mean baseline response rate. Data points superimposed over this line are the response rates for the final session of Conditioning Phase 2 calculated as a percentage of the mean response rate for the phase. In those blocks containing fewer than five (Conditioning Phase 2) or eight (Extinction phase) data points, technical problems resulted in lost data for those sessions. Note that only the first baseline (VR) is identified. All of the panels without labels are subsequent baseline conditions. 


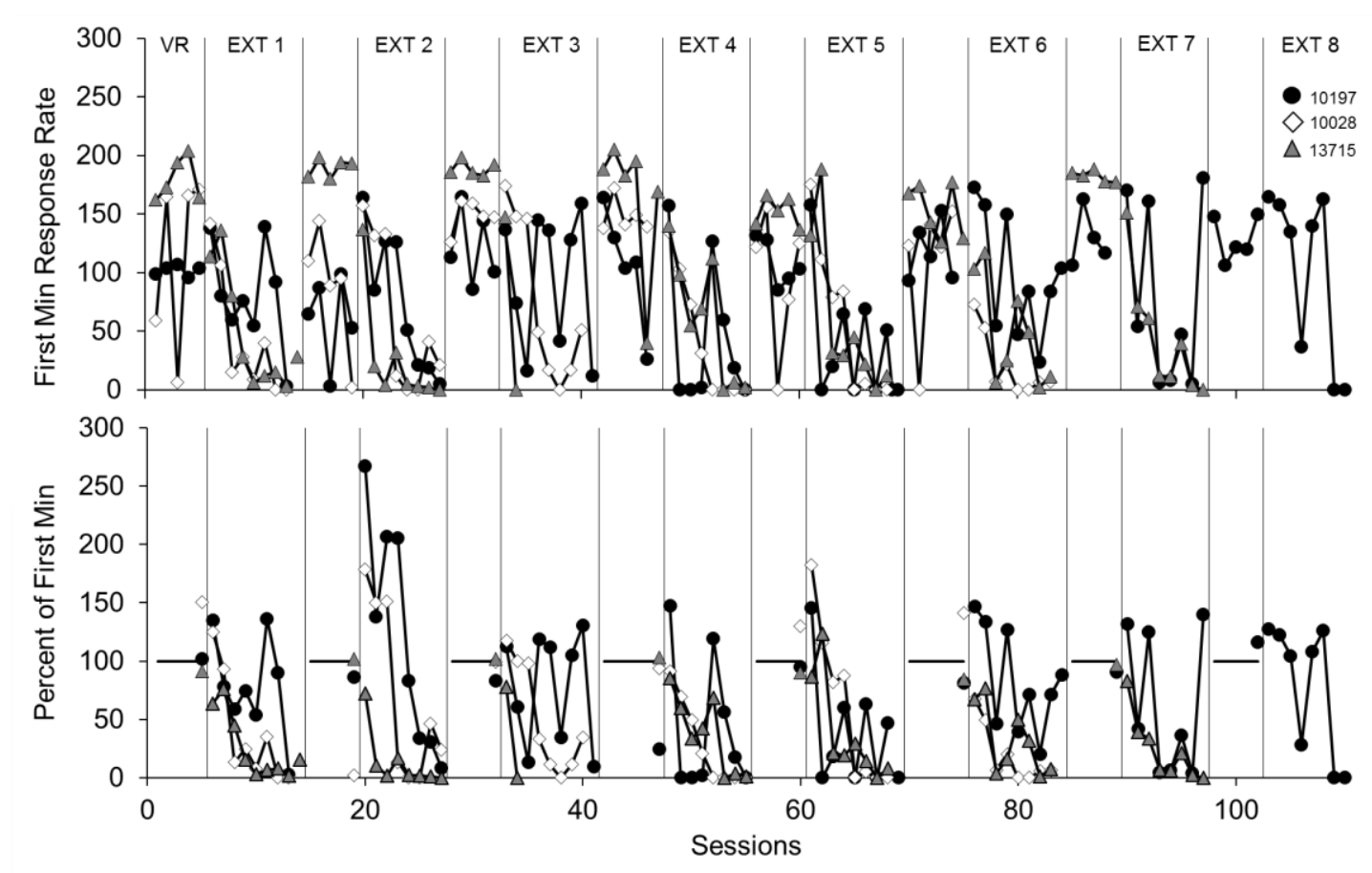

Figure 6. Response rates for each pigeon across all blocks of within-session transitions at the first-minute level of analysis cf. the start of Conditioning Phase 2 sessions. The upper panel shows the absolute response rate per minute for the sessions shown. The lower panel shows the upper-panel data transformed to a percentage of the mean response rate of each block of Conditioning Phase 2. Solid horizontal lines in Conditioning Phase 2 sections of the lower panel show the mean baseline response rate. Data points superimposed over this line are the response rates for the final session of Conditioning Phase 2 calculated as a percentage of the mean response rate for the phase. In those blocks containing fewer than five (Conditioning Phase 2) or eight (Extinction phase) data points, technical problems resulted in lost data for those sessions. Note that only the first baseline (VR) is identified. All of the panels without labels are subsequent baseline conditions. 


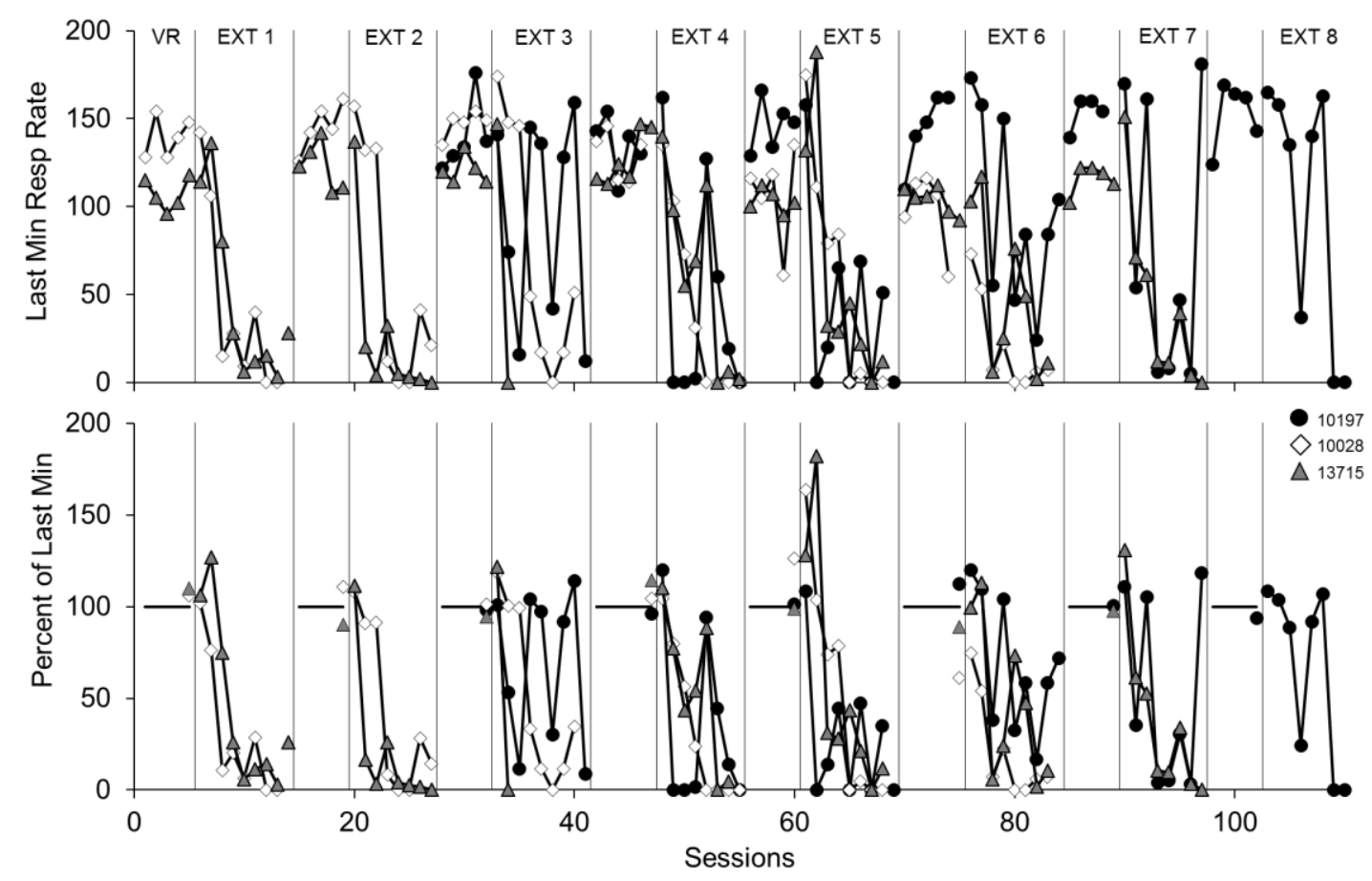

Figure 7. Response rates for each pigeon across all blocks of within-session transitions at the first-minute level of analysis $\mathrm{cf}$. the end of Conditioning Phase 2 sessions. The upper panel shows the absolute response rate per minute for the sessions shown. The lower panel shows the upper-panel data transformed to a percentage of the mean response rate of each block of Conditioning Phase 2. Solid horizontal lines in Conditioning Phase 2 sections of the lower panel show the mean baseline response rate. Data points superimposed over this line are the response rates for the final session of Conditioning Phase 2 calculated as a percentage of the mean response rate for the phase. In those blocks containing fewer than five (Conditioning Phase 2) or eight (Extinction phase) data points, technical problems resulted in lost data for those sessions. Note that only the first baseline (VR) is identified. All of the panels without labels are subsequent baseline conditions. 
rates for each block of Conditioning Phase 2.

Restricting analyses to responses during the first minute of both blocks of Conditioning Phase 2 and the Extinction phase revealed a single extinction burst during the first exposure to extinction, for 10028 (see Table 4). As in Experiment 1, the extinction burst was an intermittent phenomenon that occurred only during certain subsequent exposures to extinction. Although the extinction burst only occurred during the first exposure to extinction, the absence of a consistent effect across subjects leaves questions concerning the reliability of the phenomenon.

\section{Within-session transitions.}

Whole-session analysis of response rates during extinction. The upper panel of Figure 5 shows absolute response rates for each pigeon across all blocks of within-session transitions to extinction. The lower panel of the figure shows these same data transformed as a percentage of the average response rate for each successive block of Conditioning Phase 2.

No extinction bursts occurred during the first block of the Extinction phase as response rates during the first extinction session were substantially lower than mean Conditioning Phase 2 response rates for all three pigeons $(27 \%, 11 \%$, and $17 \%$ of mean Conditioning Phase 2 response rates for 10197, 10028, and 13715 respectively). Similar results were obtained for comparisons to maximum Conditioning Phase 2 response rates $(24 \%, 10 \%$, and $16 \%$ of maximum Conditioning Phase 2 response rates for 10197, 10028, and 13715 respectively). Subsequent exposures to the Extinction phase also did not produce extinction bursts.

First-minute analysis of response rates during extinction cf. start. Figure 6 shows response rates for each pigeon across all blocks of within-session transitions to extinction, calculated only for responses within the first minute ("start") of either Conditioning Phase 2 or the Extinction phase (depending on the session). As with previous figures, the upper panel 


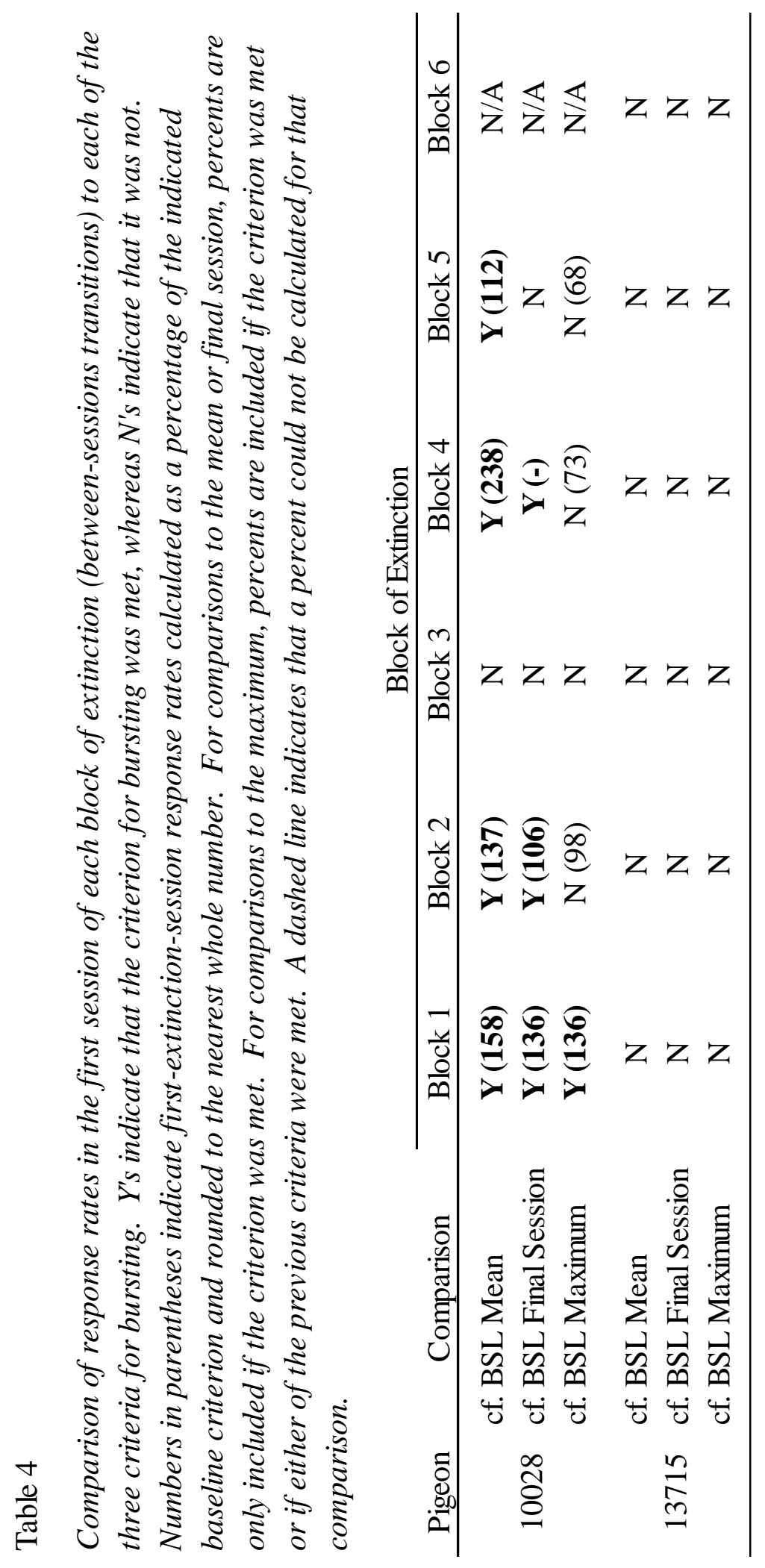


shows absolute response rates in Conditioning Phase 2 and Extinction phase sessions, whereas response rates in the lower panel were transformed to a percentage of average response rates for each block of Conditioning Phase 2.

When analyses were restricted to responses within the first minute of both Conditioning Phase 2 and the Extinction phase, extinction bursts occurred in the first session of extinction for two of the three pigeons (10197 and 10028; see Table 5). Additional bursts occurred across subsequent exposures to the Extinction phase for these two pigeons only; extinction bursts did not occur during any exposures to extinction for the third pigeon (13175). Although multiple extinction bursts occurred throughout the repeated-extinctions procedure for two pigeons, the frequency, timing, and magnitude of these bursts differed between the two pigeons. Additional differences in the timing and magnitude of these bursts were observed across successive extinction bursts within individual pigeons. As such, there was again no systematic pattern across different instances of the extinction burst to serve as a means of prediction for when the phenomenon would occur and how pronounced it would be.

First-minute analysis of response rates during extinction cf. end. The results described in the previous section compared responses in the first minute of Conditioning Phase 2 to those in the first minute of the Extinction phase. Analyses were restricted to the first minute of each session to prevent any instances of bursting during Extinction phase sessions from being "washed out" by lower response rates late in extinction. For this to serve as an accurate level of analysis, response rates within the first minute of a Conditioning Phase 2 session must be representative of those throughout the entire session. Warm-up effects or disproportionately long latencies to initiating responding during Conditioning Phase 2 sessions however, may have contributed to first-minute response rates that were not necessarily representative of those for 


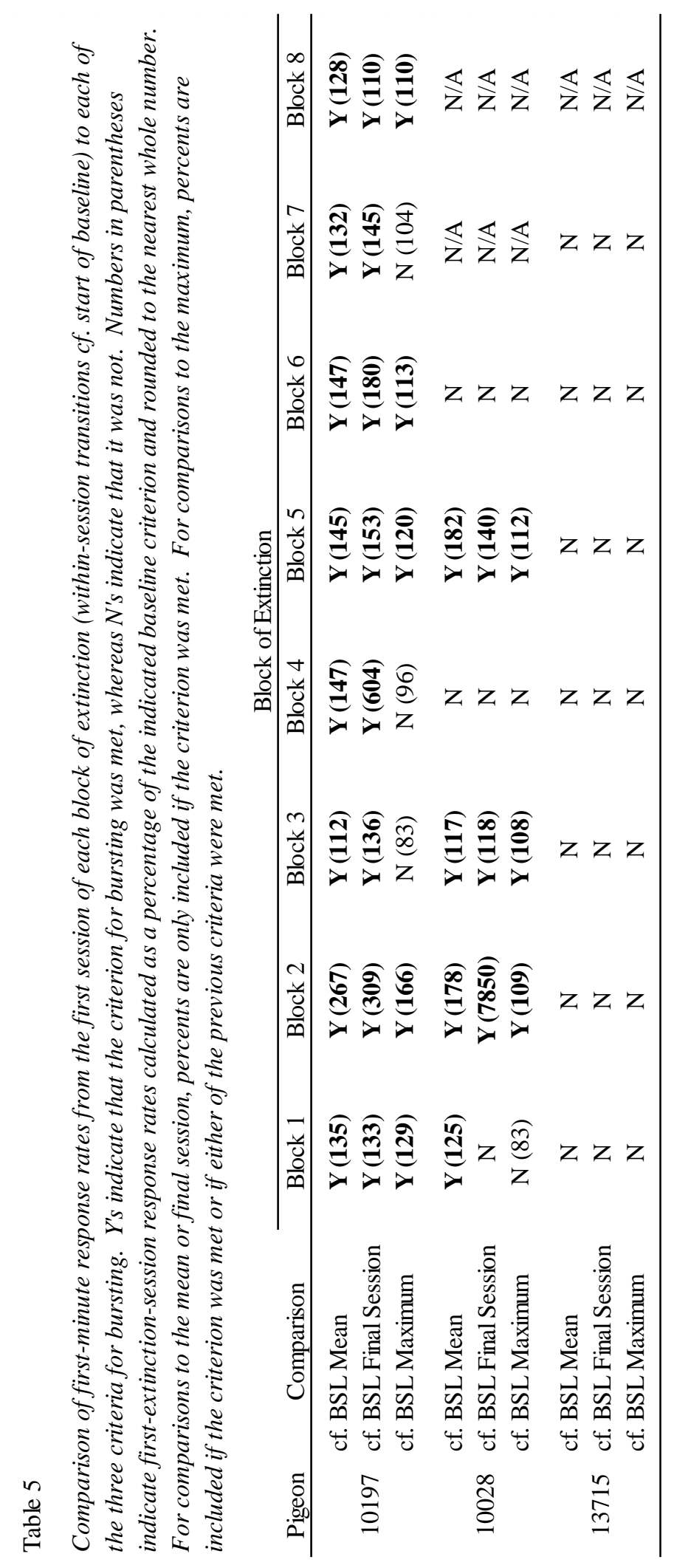


the entire session. To avoid these complications, additional comparisons were made between responses within the first minute of each Extinction phase session and the last minute ("end") of each Conditioning Phase 2 session. These comparisons were only conducted for within-session transitions to extinction due to the immediate contiguity between the end of the last session of Conditioning Phase 2 and the beginning of the first session of the Extinction phase.

The results of this analysis are shown in Figure 7. The upper panel shows absolute response rates for each pigeon across all blocks of within-session transitions to extinction, calculated only for responses within the last minute of Conditioning Phase 2 or the first minute of the Extinction phase (depending on the session). Response rates in the lower panel were transformed to a percentage of average response rates for each block of Conditioning Phase 2. Although extinction bursts occurred in the first exposure to extinction when comparing response rates in the first minute of extinction to the first minute of Conditioning Phase 2 (see Figure 6), no extinction bursts occurred when the comparison was instead between response rates in the first minute of extinction and the final minute of Conditioning Phase 2. Extinction bursts did occur during subsequent exposures to the Extinction phase (see Table 6), although the blocks of extinction in which they occurred were not always the same as those identified in the previous analysis. Despite these similarities between pigeons, unsystematic differences again were observed in the frequency, timing, and magnitude of bursts across subjects. Also, idiosyncratic dimensions of bursting again were observed across successive bursts within individual pigeons. For all three pigeons, extinction bursts occurred during certain exposures to extinction and not others, with differing magnitudes across instances. As such, the extinction burst was an intermittent phenomenon that was present in certain exposures to extinction and not others, without consistent dimensions across occurrences. 


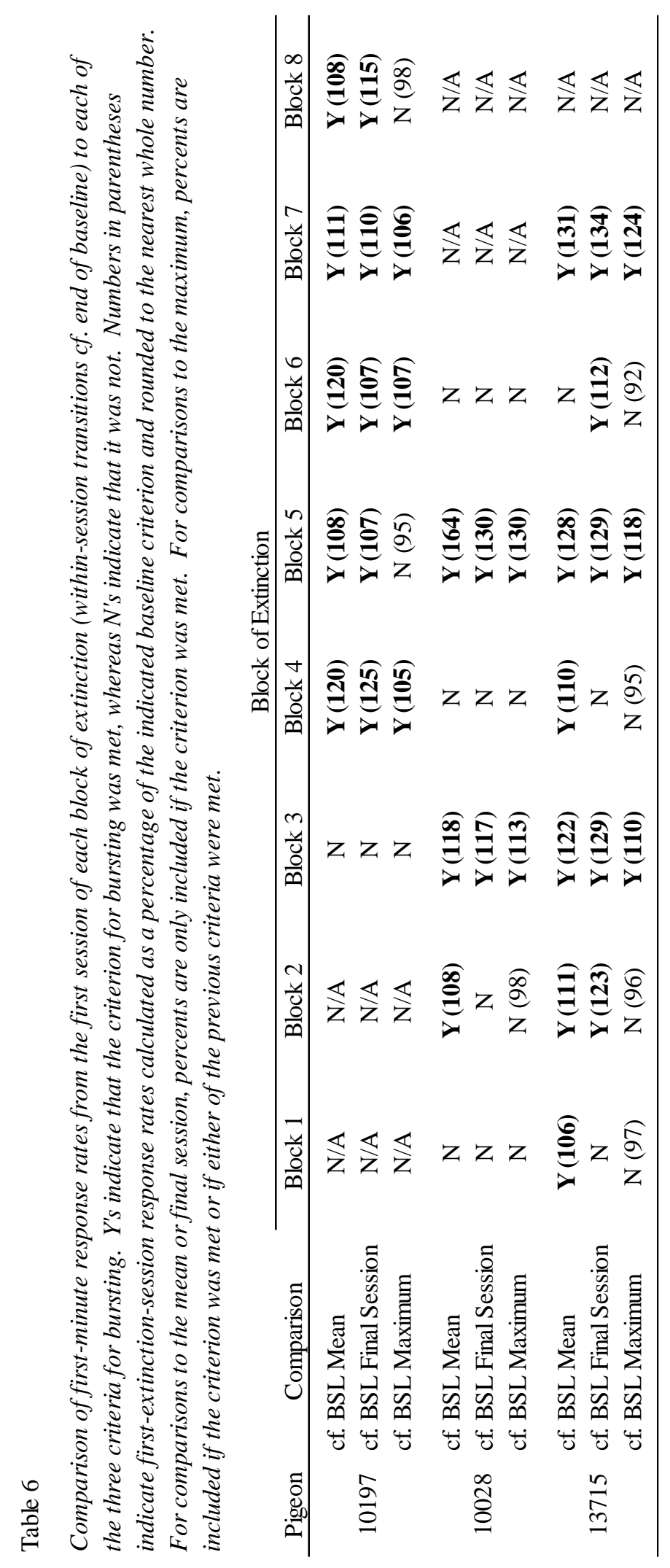


Post-reinforcement pause analysis. It is possible that the increases in response rate early during extinction detailed in the previous sections are not evidence of an actual behavioral phenomenon but are instead simply an artifact produced by the absence of reinforcer delivery during Extinction phase sessions. During Conditioning Phase 2 sessions in which reinforcers were delivered on a VR 20 schedule, post-reinforcement pauses (PRPs) occurred after every food delivery. Conversely, during Extinction phase sessions in which no reinforcers are delivered, PRPs consequently do not occur. Including PRPs in response rate calculations for Conditioning Phase 2 sessions decreases the response rate, and thus may be responsible for the comparative increase in response rate for Extinction phase sessions (and thus the extinction burst).

To eliminate this influence and determine if the extinction bursts described above were artifacts of the inclusion of PRPs in Conditioning Phase 2 response rates, additional analyses were conducted comparing run rates for Conditioning Phase 2 sessions to those of Extinction phase sessions. As such, the total PRP time for each Conditioning Phase 2 sessions was excluded when calculating run rates of responding. Although these calculations were performed at all three levels of analysis (whole-session, first-minute cf. start, and first-minute cf. end), systematic effects were not observed at the whole-session level, and thus are not included below.

Run rates for all Conditioning Phase 2 sessions were substantially higher than overall response rates. Consequently, there was a substantial decrease in first-extinction-session run rates calculated as a percentage of baseline across all exposures to extinction (see Tables 7and 8). Assessed in this way, only a single extinction bursts occurred. Notably, extinction bursts did not occur even in blocks of extinction in which they had occurred according to previous analyses that included PRP time. These results suggest that the extinction bursts detailed in the previous sections simply may be an artifact of the extinction procedure - namely the cessation of 


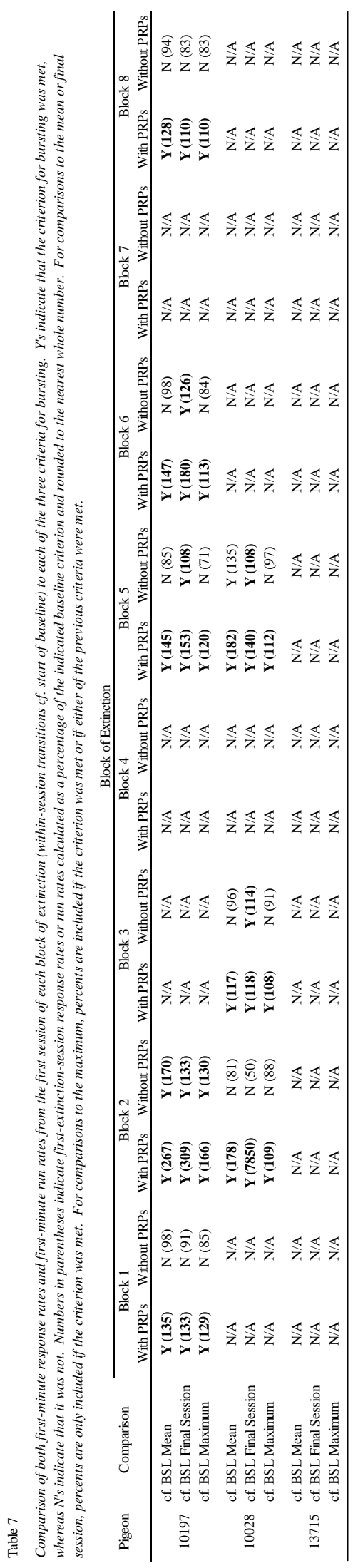




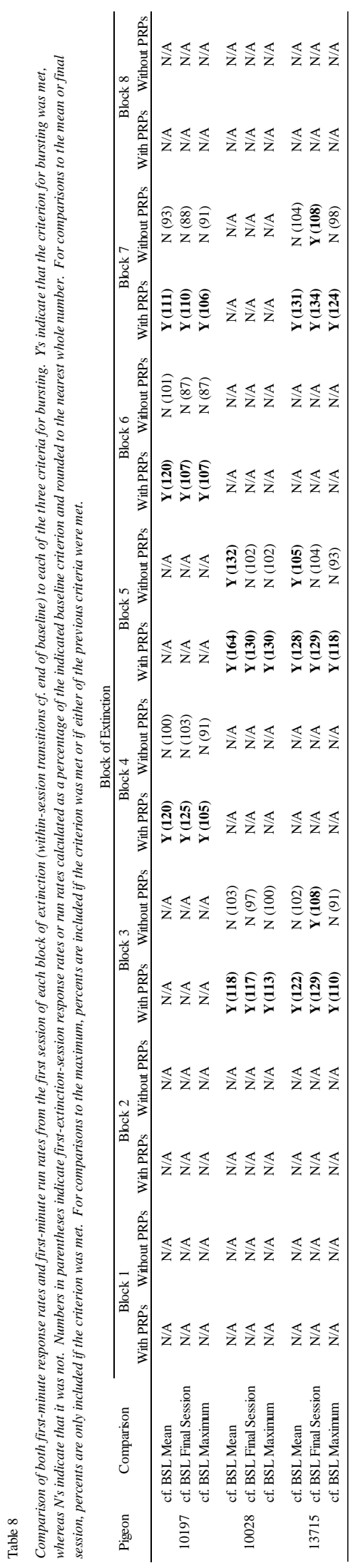


reinforcer deliveries and thus the absence of PRPs - as opposed to an actual increase in response rate compared to baseline.

\section{Discussion}

Successive alternations between blocks of a conventional VR schedule of conditioning and blocks of extinction intermittently produced extinction bursts in three pigeons. As was reported with an increasing VR schedule, extinction bursts did not occur when analyses included responses from throughout entire conditioning and extinction sessions. Instead, extinction bursts only occurred when analyses were restricted to responses within the first minute of extinction sessions and either the first or last minute of conditioning sessions (i.e. cf. start and cf. end). Although bursts occurred regardless of the type of transition between blocks of conditioning and extinction (i.e. between-sessions or within-session), bursts were more common following withinsession transitions to extinction. The occurrence, timing, and magnitude of these bursts was inconsistent both within and between subjects. Each pigeon exhibited different frequencies, magnitudes, and times of occurrence of bursting throughout the repeated extinctions procedure. Additionally, these dimensions of bursting were not constant in those pigeons exhibiting extinction burst during more than a single block of extinction.

Measurement considerations. Several details of the repeated-extinctions procedure that have implications for the measurement of the extinction burst were identified in Experiment 1. The adjustments to the procedure made in Experiment 2 introduced three additional measurement considerations that should be addressed: the type of transition from baseline to extinction, the inclusion of PRPs in response rate calculations, and the comparison of response rates from the first minute of extinction to the final minute of baseline. As different types of transitions and the inclusion of the PRP were only analyzed in Experiment 2, they will be 
addressed here. Conversely, since responses in the first minute of extinction were compared to responses in the final minute of baseline in Experiment 3 as well, discussion of this analysis will be saved for the General Discussion section.

Effects of transition type. Although extinction bursts occurred for both types of transitions to extinction, substantially more bursts occurred following within-session transitions to extinction than between-sessions transitions. As the extinction burst is often defined as a transient phenomenon (e.g. Cooper et al., 2007; Domjan \& Burkhard, 1982; Reynolds, 1968), this finding could be explained as a consequence of decreases in the magnitude of the burst across repeated exposures to extinction. Given, however, that all blocks of within-session transitions to extinction were conducted after all blocks of between-sessions transitions to extinction, this explanation cannot account for the obtained results. Thus, the lower frequency of extinction bursts following between-sessions transition to extinction appears to represent a fundamental difference between the two types of transitions as opposed to sequencing effects.

One difference between the two types of transitions is the discriminability of the onset of extinction. For between-sessions transitions, several discriminative stimuli were present immediately prior to both Conditioning Phase 2 and Extinction phase sessions. Before sessions in both phases, each pigeon was removed from its home cage, transported to and placed inside the operant chamber, and subjected to a 3-min blackout before the keylight turned on and the session began. Conversely, for the within-session transitions to extinction, the onset of the first session of extinction immediately followed the end of the final session of Conditioning Phase 2 without the presentation of the same stimuli as during baseline. As such, the onset of extinction was more discriminable following within-session transitions to extinction than between-sessions transitions. Conclusions about the effect of this difference in discriminability are supported by 
how only signaled within-session changes in reinforcement ratios and not unsignaled changes induced corresponding changes in performance on conditional discrimination procedures (Ward \& Odum, 2008). Thus, changes in the discriminability of the transition to extinction across types of transitions may have differentially influenced response rates early in extinction. Given this effect, consideration should be given to the type of transition between baseline and extinction sessions in future experiments on the nature of the extinction burst.

Effects of the inclusion of post-reinforcement-pauses. When responding during extinction was evaluated by comparing run rates from the first session of extinction to run rates from Conditioning Phase 2, only one extinction burst was observed. This suggests that the increases in response rate observed during extinction that have classically been referred to as extinction bursts are in fact artifacts of the absence of PRPs during extinction. Because these pauses do not occur during extinction, response rate calculations produce comparatively higher response rates than those obtained during preceding baseline sessions, giving the illusion of a burst in response rate.

This difference in the frequency of the extinction burst across the two manners of calculation raises several questions about how increases in responding at the onset of extinction should be defined, measured, and conceptualized. In both meta-analyses cited previously, the extinction burst was simply defined as an increase in responding, without any specification of whether this was measured via changes in response rate, run rate, or the absolute number of responses (Lerman \& Iwata, 1995; Lerman et al., 1999). As the method of calculation can influence the number of instances of the extinction burst, the results of these meta-analyses concerning the frequency of the phenomenon should be interpreted cautiously. The relative number of individual experiments within the meta-analyses that measured behavior in extinction 
via either changes in response rate, run rate, or the absolute number of responses may have heavily influenced the frequency of the extinction burst reported by the authors. In the future, when attempting to assess the frequency of the extinction burst, care should be taken to ensure that the phenomenon is measured in a consistent manner across experiments. Furthermore, additional investigation of the extinction burst as defined by changes in run rates is warranted to assess the generality of this finding.

Constraints on generality. Given the implications of this finding on the nature of the extinction burst, additional comparisons between bursts measured via changes in response rates and changes in run rates are necessary. Currently however this analysis was conducted only for the bursts that occurred in Experiment 2. Given the constraints limits on generality imposed by the increasing VR schedule of reinforcement used in Experiment 1, it was decided that an analysis of run rates would be more prudent when a conventional VR schedule was used instead during baseline sessions.

Although the use of a conventional VR schedule addressed one of the constraints with the schedule used in Experiment 1 and allowed for the analysis of run rates, there are still limitations associated with the use of a VR schedule. Due to the variable nature of the schedule, it was still difficult to identify the exact point of contact with the extinction contingency and thus specify the point after which responses would count as instances of bursting. This was addressed in Experiment 3 by substituting for the VR schedule an FR 1 schedule of conditioning during baseline conditioning sessions, allowing for an analysis of the extinction burst in which the point of contact with the extinction contingency is precisely specified. 


\section{Experiment 3}

Even though Experiment 2 addressed some of the concerns associated with the procedure of Experiment 1, the same issues associated with the use of a VR schedule during Conditioning Phase 2 were present. Furthermore, as noted in the introduction, unlike the present Experiments 1 and 2, the extinction burst most frequently has been studied following a transition from a schedule of continuous reinforcement to extinction. Thus, in Experiment 3 transitions from FR 1 to extinction were examined.

\section{Procedure}

Conditioning Phase 1. The combined autoshaping and FR sessions were conducted as described in the General Method section.

Conditioning Phase 2. Responding was maintained by an FR 1 schedule such that each response produced 3-s access to food. All other aspects of the phase were conducted as described in the General Method section.

Extinction. Keypecking was extinguished across eight sessions as described in the General Method section, with each such session lasting for 10 minutes. After the eighth extinction session, the three-phase sequence was repeated at least 5 times, as detailed in Table 9 . In Experiment 3 all transitions from Conditioning Phase 2 to the Extinction phase were conducted within session.

\section{Results}

Time-course of extinction. For each pigeon, repeated exposures to extinction produced behavioral effects consistent with those observed in Experiments 1 and 2 (see Figures 8 and 9). Response rates during Extinction phase sessions decreased across successive blocks of extinction, across successive sessions within a block, and over time within individual sessions. 
Table 9

Number of Transitions For Each Pigeon

in Experiment 3

\begin{tabular}{cc} 
Pigeon & Number of Transitions \\
\hline 20542 & 6 \\
18390 & 7 \\
1576 & 5 \\
\hline
\end{tabular}




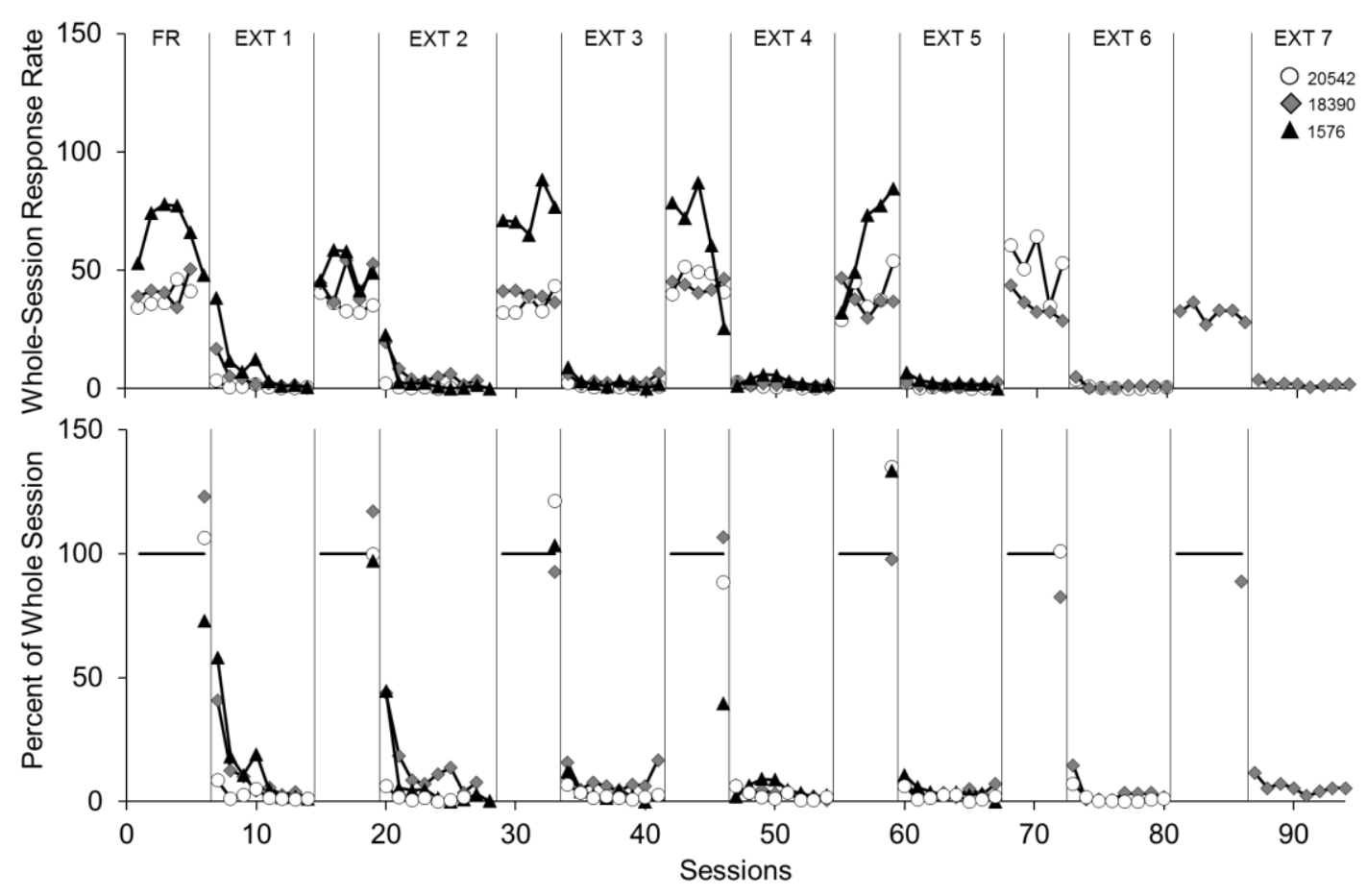

Figure 8. Response rates for each pigeon across blocks of the repeated-extinction procedure at the whole-session level of analysis. The upper panel shows the absolute response rate per minute for the sessions shown. The lower panel shows the upper-panel data transformed to a percentage of the mean response rate of each block of Conditioning Phase 2. Solid horizontal lines in Conditioning Phase 2 sections of the lower panel show the mean baseline response rate. Data points superimposed over this line are the response rates for the final session of Conditioning Phase 2 calculated as a percentage of the mean response rate for the phase. In those blocks containing fewer than five (Conditioning Phase 2) or eight (Extinction phase) data points, technical problems resulted in lost data for those sessions. Note that only the first baseline (FR) is identified. All of the panels without labels are subsequent baseline conditions. 


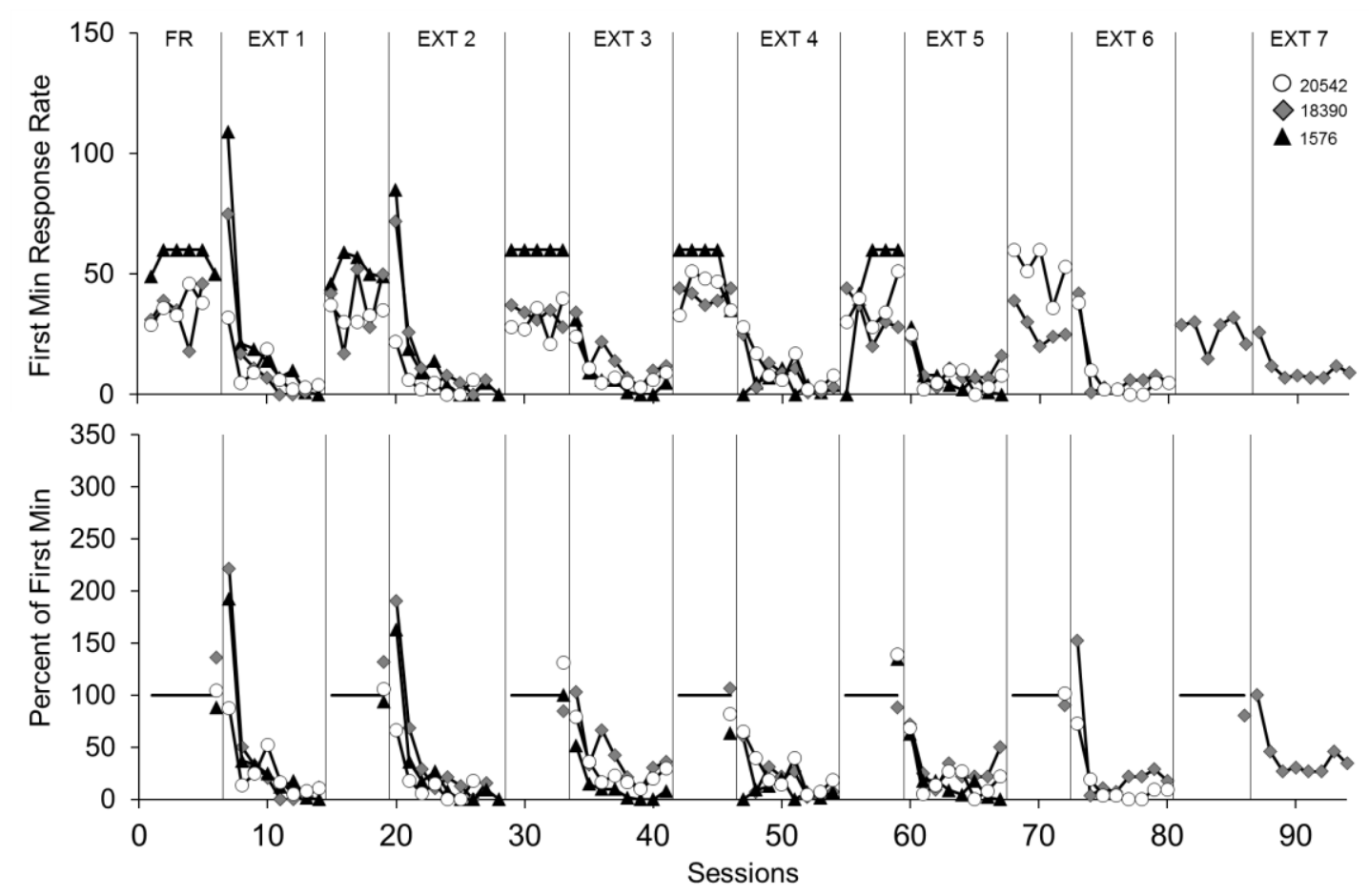

Figure 9. Response rates for each pigeon across all blocks of the repeated-extinction procedure at the first-minute level of analysis cf. the start of Conditioning Phase 2 sessions. The upper panel shows the absolute response rate per minute for the sessions shown. The lower panel shows the upper-panel data transformed to a percentage of the mean response rate of each block of Conditioning Phase 2. Solid horizontal lines in Conditioning Phase 2 sections of the lower panel show the mean baseline response rate. Data points superimposed over this line are the response rates for the final session of Conditioning Phase 2 calculated as a percentage of the mean response rate for the phase. In those blocks containing fewer than five (Conditioning Phase 2) or eight (Extinction phase) data points, technical problems resulted in lost data for those sessions. Note that only the first baseline (FR) is identified. All of the panels without labels are subsequent baseline conditions. 
Whole-session analysis of response rates during extinction. Absolute response rates for each pigeon across all blocks of the experiment are shown in the upper panel of Figure 8. As in previous figures, response rates in the lower panel were calculated as a percentage of the average response rate for each block of Conditioning Phase 2.

Extinction bursts did not occur during the first block of the Extinction phase for any of the three pigeons. Response rates during the first session of the Extinction phase were substantially less than mean Conditioning Phase 2 response rates for all three pigeons (9\%, 41\%, and 58\% of mean Conditioning Phase 2 response rates for 20542, 18390, and 1576 respectively). Similar results were obtained for comparisons to maximum Conditioning Phase 2 response rates (7\%, 33\%, and 49\% of mean Conditioning Phase 2 response rates for 20542, 18390, and 1576 respectively). Additionally, there were no instances of bursting across any subsequent exposures to extinction.

First-minute analysis of response rates during extinction cf. start. Figure 9 shows response rates for each pigeon across all blocks of the experiment, calculated only for responses within the first minute (start) of either Conditioning Phase 2 or the Extinction phase (depending on the session). Absolute response rates are shown in the upper panel of the figure. Response rates in the lower panel were transformed to a percentage of average response rates for each block of Conditioning Phase 2.

Extinction bursts occurred in the first session of extinction for two of the three pigeons (18390 and 1576; see Table 10) when calculations only included responses from the first minute of each session. For both pigeons, similar results were obtained across the following four exposures to the extinction phase. In the second block of extinction, a second extinction burst smaller in magnitude compared to that of the preceding block - occurred. Subsequently, during 


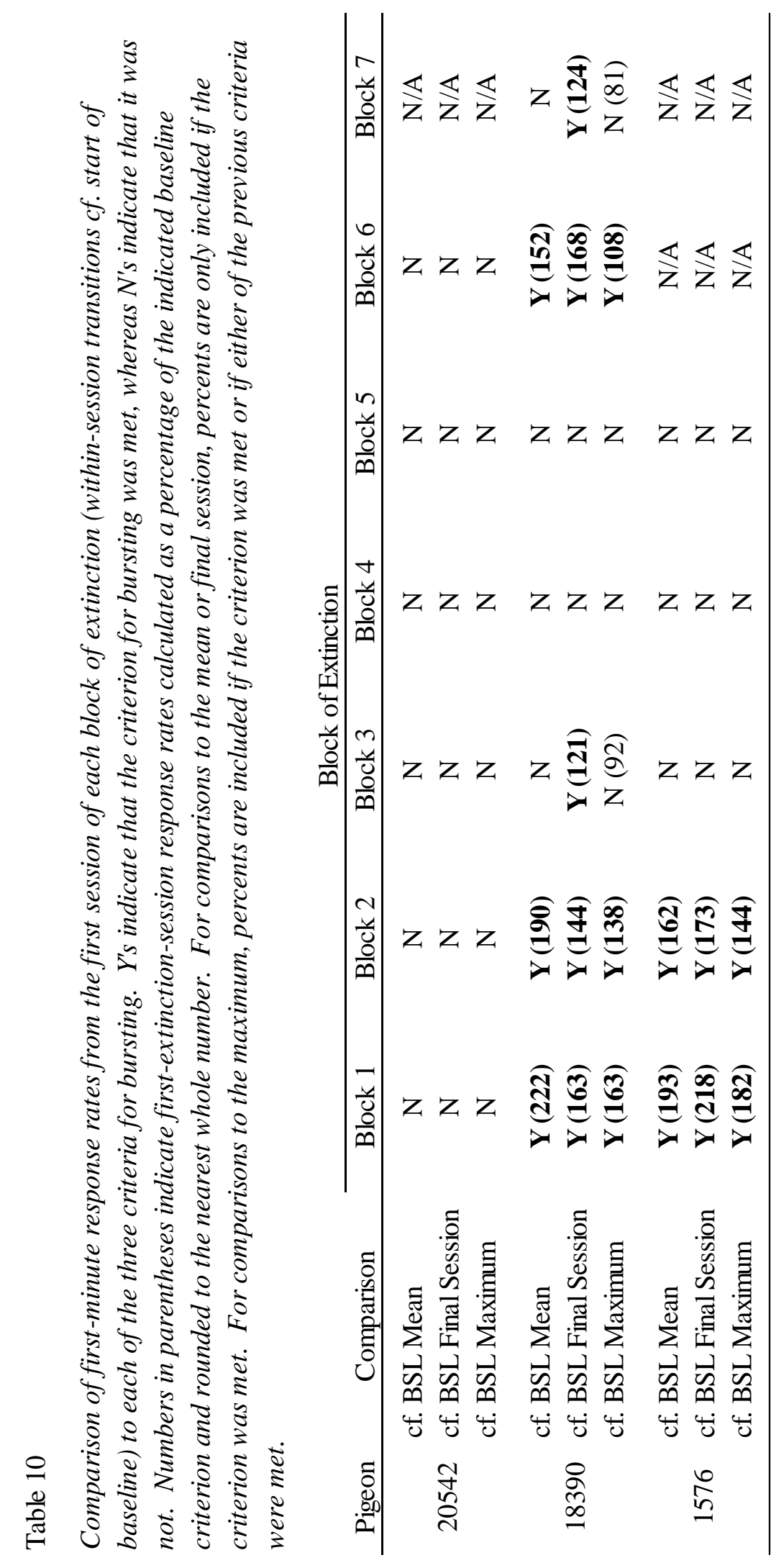


the third, fourth, and fifth blocks of extinction, extinction bursts did not occur. Furthermore, response rates during the first session of each block of extinction decreased across the first four exposures to extinction. Extinction bursts did not occur during any exposures to extinction for the third pigeon (20542).

Despite these similarities across the first five blocks of the experiment, there were two differences in the dimensions of bursting between the two pigeons. First, the magnitude of both initial extinction bursts was substantially higher for 18390 than for 1576 . Second, a third extinction burst occurred for 18390 in the sixth exposure to extinction. The absence of a fourth extinction burst during the seventh exposure to extinction suggests this was not a persistent recurrence of the phenomenon, but this remains to be confirmed by an eventual sixth exposure to extinction for 1576 .

First-minute analysis of response rates during extinction cf. end. Figure 10 shows response rates for each pigeon across all blocks of the experiment, calculated only for responses within the final minute (end) of Conditioning Phase 2 sessions and the first minute of Extinction phase sessions. Absolute response rates are shown in the upper panel of the figure, whereas those in the lower panel were transformed to a percentage of average response rates for each block of Conditioning Phase 2.

Extinction bursts occurred in the first session of extinction for two of the three pigeons (18390 and 1576; see Table 11). As with the previously described analyses, similar patterns of bursting occurred across subsequent exposures to the Extinction phase for both pigeons. A second extinction burst occurred in the second block of extinction, and no other bursts occurred across the remaining blocks. This second extinction burst, again, was smaller in magnitude than the burst that occurred in the first block. Extinction bursts did not occur during any exposures to 


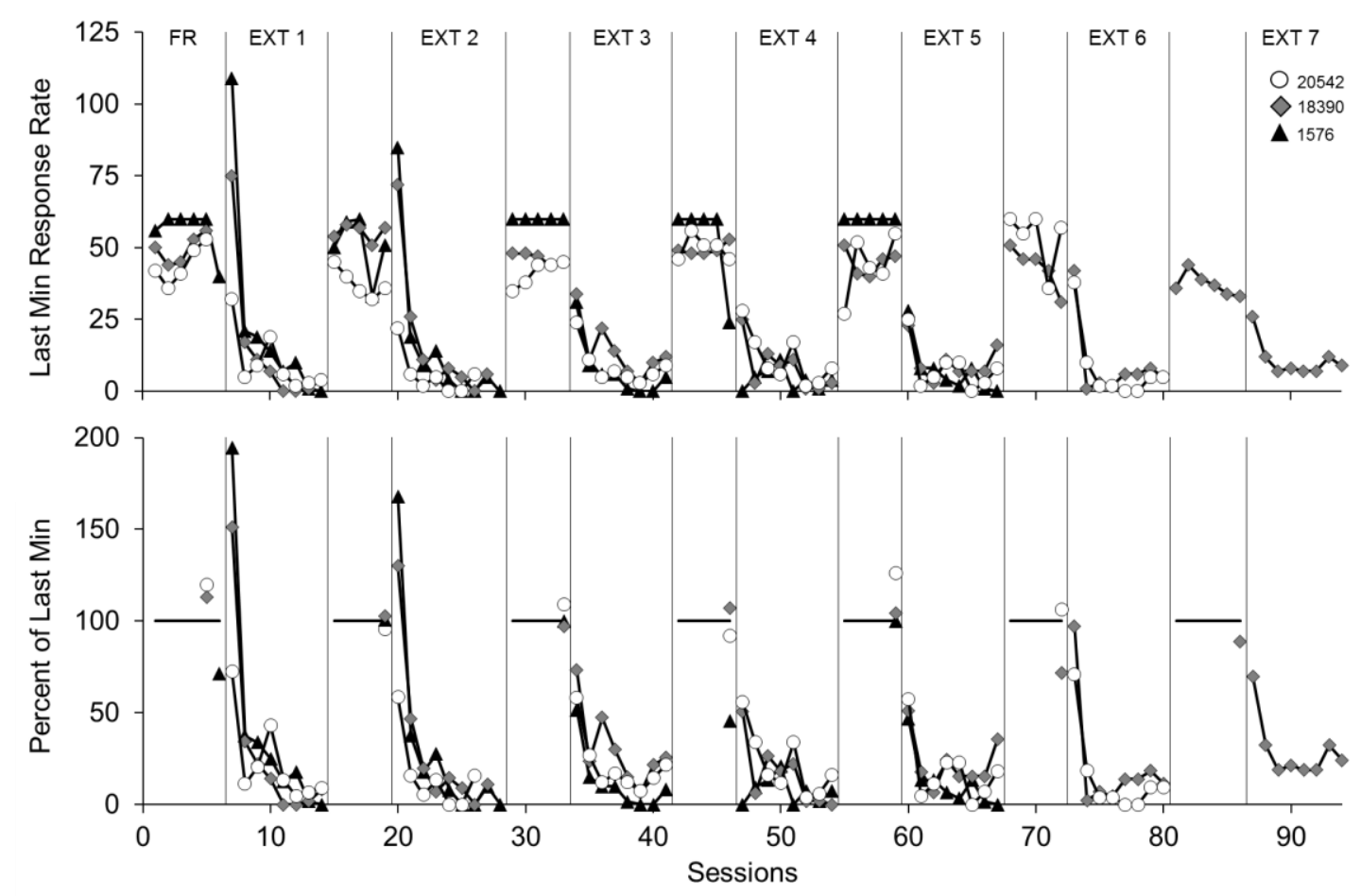

Figure 10. Response rates for each pigeon across all blocks of the repeated-extinction procedure at the first-minute level of analysis cf. the end of Conditioning Phase 2 sessions. The upper panel shows the absolute response rate per minute for the sessions shown. The lower panel shows the upper-panel data transformed to a percentage of the mean response rate of each block of Conditioning Phase 2. Solid horizontal lines in Conditioning Phase 2 sections of the lower panel show the mean baseline response rate. Data points superimposed over this line are the response rates for the final session of Conditioning Phase 2 calculated as a percentage of the mean response rate for the phase. In those blocks containing fewer than five (Conditioning Phase 2) or eight (Extinction phase) data points, technical problems resulted in lost data for those sessions. Note that only the first baseline (FR) is identified. All of the panels without labels are subsequent baseline conditions. 


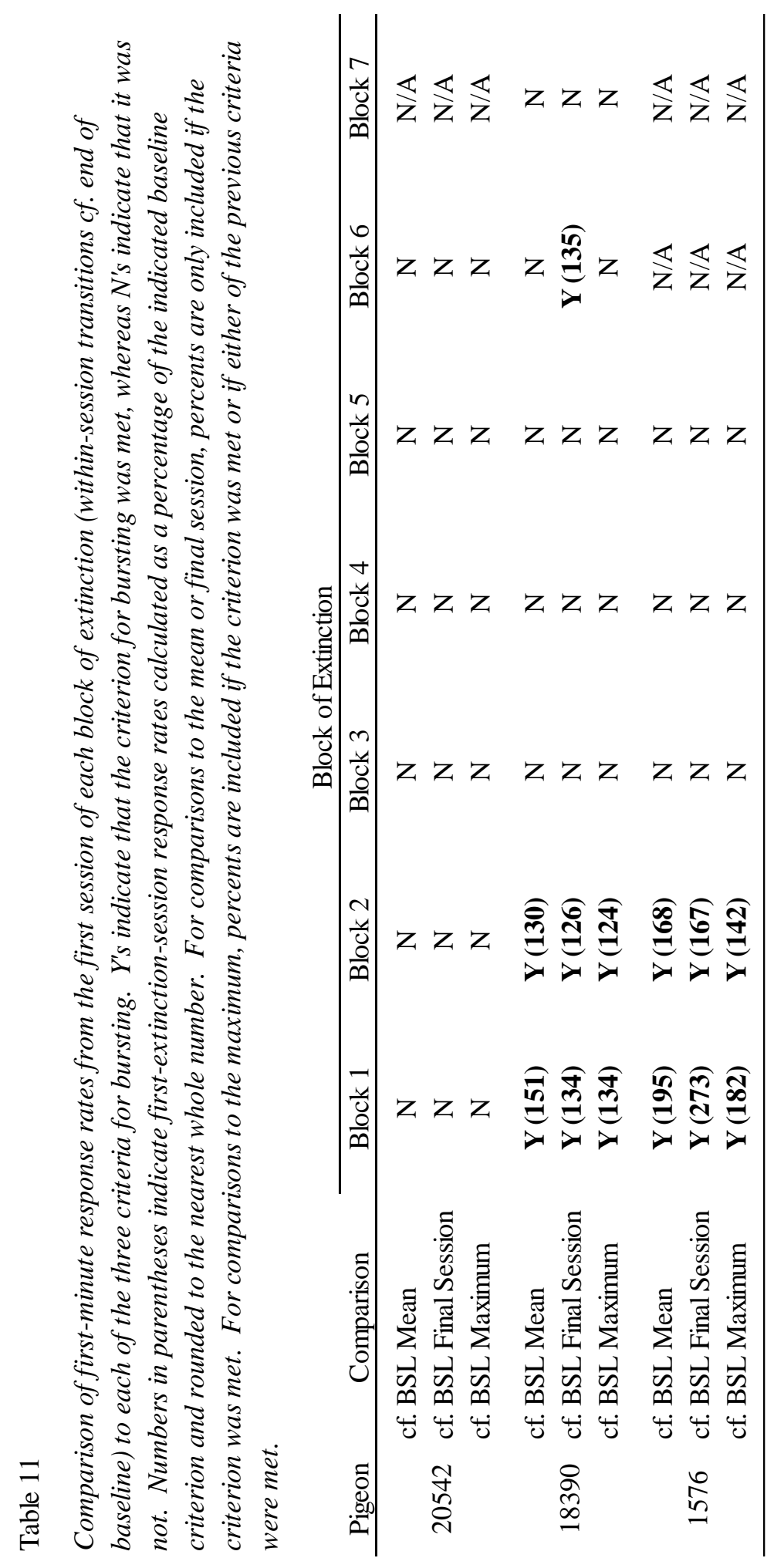


extinction for the third pigeon. In sum, the extinction burst was again a transient phenomenon, but with a consistent and predictable pattern of occurrence in those pigeons that it did occur. Extinction bursts reliably occurred in the first two exposures to extinction, with a decreasing magnitude across successive occurrences.

\section{Discussion}

Repeated alternations between blocks of an FR 1 schedule of conditioning and blocks of extinction produced extinction bursts during early blocks of extinction in two of three pigeons. These extinction bursts were observed only when analyses were restricted to include only responses within a one-minute interval during Conditioning Phase 2 and Extinction phase sessions. For both pigeons, the magnitude of the extinction burst decreased across successive blocks of extinction and disappeared by the third block of extinction. For one pigeon, a third burst occurred in the sixth block of extinction only when analyses involved comparisons between responses in the first minute of Conditioning Phase 2 and Extinction.

Effects of behavioral history. At the first-minute level of analysis, extinction bursts occurred for only two of the three pigeons. The absence of extinction bursts during the first two exposures to the Extinction phase for Pigeon 20542 may be a function of its prior history of repeated extinctions. Before exposure to repeated within-session transitions to extinction following an FR 1 schedule of conditioning, this pigeon was subjected to six exposures to between-sessions transitions to extinction interspersed with FR 1 schedules of conditioning. As these between-session transitions were only conducted for one pigeon, the data are not reported here. Conversely, the other two pigeons were experimentally naïve before beginning Experiment 3 and thus did not have this history of repeated extinctions. The absence of extinction bursts in the one pigeon with prior exposure to the repeated-extinctions procedure suggests that the 
extinction burst is a transient phenomenon that does not persist across repeated exposures to extinction. This is supported by the disappearance of the extinction burst upon the third transition to extinction for the other two, previously experimentally naïve, pigeons.

Constraints on generality. Although the use of an FR 1 schedule of reinforcement addresses the major considerations regarding generality discussed in Experiments 1 and 2, there are other constraints associated with continuous reinforcement. The main shortcoming with using an FR 1 schedule during Conditioning Phase 2 is associated with the PRP analysis described in Experiment 2. As discussed, this analysis suggested that the increases in firstsession response rates that occurred blocks of extinction during Experiment 2 were not evidence of extinction bursts but were simply artifacts of the elimination of reinforcer delivery, and thus PRPs, associated with extinction. As PRPs cannot be eliminated from consideration in schedules of continuous reinforcement, this possibility could not be investigated any further in Experiment 3. Thus, additional investigation to determine if the elimination of PRPs is definitively responsible for what has been termed the extinction burst is required.

Furthermore, the effects of behavioral history described above limit the conclusions that can be drawn from the present findings. The inclusion of additional, experimentally naïve pigeons and an investigation of dimensions of bursting following repeated exposures to extinction and FR 1 conditioning sessions would help to answer this question and provide a more complete analysis of bursting.

\section{General Discussion}

Across three experiments, successive alternations between blocks of conditioning and extinction intermittently produced extinction bursts when analyses included responses from only the first minute of Extinction phase sessions. Each pigeon exhibited different frequencies, 
magnitudes, and times of occurrence of bursts throughout the repeated-extinctions procedure. Additionally, these dimensions of bursting were not constant across repeated bursts within individual pigeons. Various details of how the burst was measured, including the schedule in effect during Conditioning Phase 2 sessions, the manner of transition to extinction, and the level of analysis, also influenced the occurrence and dimensions of the extinction burst.

\section{Measurement Considerations}

Whole-session and first-minute analysis. Extinction bursts occurred only when response rates in the first minute of Extinction phase sessions were considered, and even then, the bursts were not systematic. As the extinction burst often is described as transient (e.g. Cooper et al., 2007; Domjan \& Burkhard, 1982; Reynolds, 1968) this observation is not surprising. Although previous assessments of the extinction burst have focused on wholesession response rates (e.g. Lerman \& Iwata, 1995; Lerman et al., 1999), it stands to reason that any instances of the phenomenon would be localized to the beginning of the extinction session given its transient nature. Following this immediate increase early in the session however, response rates would begin to decline and eventually reach zero (Cooper et al., 2007). Thus, depending on the duration of the extinction session, any instances of bursting at the wholesession level of analysis could be "washed out" by including in the analysis extended periods late in extinction during which responding had ceased. In the present series of experiments, the longest sessions of extinction were the 20-min sessions of Experiment 3. Thus, the complete absence of bursts at the whole-session level of analysis across all three experiments underscores the transience of the phenomenon.

The first-minute analysis is a novel method for investigating the occurrence of the extinction burst. As such, it is necessary to address potential misgivings that may be expressed 
about the merits of the analysis. One such concern is whether reinforcers were delivered in the first minute of a Conditioning Phase 2 session, and thus whether the extinction contingency was contacted in the first minute of an Extinction phase session. Average first-extinction-session response rates were substantially higher than the highest ratio requirement in each of the schedules in effect at the beginning of Conditioning Phase 2 sessions. As such, each pigeon should have earned several reinforcers during the first minute of Conditioning Phase 2 sessions and contacted the extinction contingency within the first minute of the first session of the Extinction phase. Thus, the first-minute level of analysis is an appropriate level for investigating the extinction burst.

Alongside reports of the extinction burst, previous assessments of overall patterns of responding throughout extinction also have focused on responding at the whole-session level of analysis (e.g. Anger \& Anger, 1976). Overall, similar general patterns of responding to those described by Anger and Anger (1976) were obtained in each of the three experiments. The conventional negatively-decelerating extinction curve (see Cooper et al., 2007) was replicated across successive extinctions for each pigeon. Throughout the repeated-extinctions procedure, response rates during Extinction-phase sessions decreased both across successive blocks of extinction and across successive sessions within an individual block. One notable difference between the results of Anger and Anger and those of the present series of experiments concerns responding in later blocks of extinction. Anger and Anger reported that in later blocks of extinction, the number of responses in sessions later in the block increased compared to those from earlier blocks of extinction. This increase in the number of responses was not observed in the current experiment. Future experiments may investigate this phenomenon to determine the 
conditions controlling the occurrence of this increase in the number of responses as extinction blocks continue.

Last-minute analysis. Although reinforcers were delivered in the first minute of each session, the potential for warm-up effects means response rates for the first minute of Conditioning Phase 2 sessions may not have been representative of responding throughout entire sessions. As such, additional comparisons between response rates from the first minute of the Extinction phase and the final minute of Conditioning Phase 2 were conducted to evaluate changes between responding during extinction and baseline more accurately.

Mean response rates for the final minute of Conditioning Phase 2 were substantially higher than mean response rates for the first minute of the phase for five of the six pigeons used in Experiments 2 or 3. Furthermore, for four of the six pigeons, response rates from the last minute of Conditioning Phase 2 were more similar to whole-session response rates than were response rates from the first minute of Conditioning Phase 2. Despite these differences in response rate across the two sampled portions of baseline, there were not substantial differences in the overall number of extinction bursts that occurred across the two analyses. There were, however, differences in the blocks of extinction in which these bursts occurred. Taken together, these effects suggest that response rates from the final minute of Conditioning Phase 2 sessions are more representative of whole-session response rates as they are not influenced by warm-up effects. As such, the last-minute analysis may prove to be a superior method to evaluate the occurrence, frequency, and dimensions of extinction bursts compared to both the whole-session and first-minute levels of analysis. Given the differences in blocks of extinction in which bursts were observed however, additional investigation concerning the differences in timing of the bursts across the two analyses should be conducted. 
Assessment of bursting criteria. Two criteria were used to define the extinction burst. First, response rates in the first session of a block of extinction had to be at least 5\% higher than either the response rate of the final session of the preceding block of Conditioning Phase 2 or the mean response rate of the phase. Second, sessions that met this criterion then were compared to the maximum response rate of the preceding block of Conditioning Phase 2. If there was at least a 5\% increase in response rate compared to the maximum of Conditioning Phase 2, then an extinction burst was said to have occurred in that block of extinction.

When identifying instances of bursting, there is a precedent for using the maximum number of responses during baseline as the standard to which to compare the number of responses during extinction (e.g., Lerman \& Iwata, 1995; Lerman et al., 1999). These comparisons, however, were conducted using responses at the whole-session level of analysis. Conversely, in the present series of experiments, extinction bursts were observed only at the oneminute level of analysis and not at the whole-session level. Additionally, response rates generally were more variable at the first-minute level of analysis than the whole-session level of analysis. Given this large degree of variability, a comparison to the maximum response rate during baseline may not be an appropriate criterion at the first-minute level of analysis as the maximum response rate was not always representative of response rates for the remaining sessions in the phase.

Instead, a comparison to just the mean response rate of the preceding phase may be more appropriate. If the extinction burst were redefined as at least a 5\% increase in the response rate of the first session of the Extinction phase compared to the average response rate during the preceding block of Conditioning Phase 2, responding in substantially more blocks of extinction across the three experiments would be classified as instances of bursting. Furthermore, the mean 
magnitude of extinction bursts that occurred according to this new criterion would be substantially higher than those that occurred according to the existing criteria that includes a comparison to the maximum. The drastic difference in the frequency and magnitude of instances of bursting across these two operational definitions underscores the sensitivity of the extinction burst to its definition and the importance of consistent, standardized criteria for bursting across experiments. Perhaps increases in the response rate of the first session of extinction exceeding the mean baseline response rate should be termed extinction bursts, whereas increases compared to the maximum baseline response rate should be classified as "extinction eruptions" (see Figure $11)$.

Transition to extinction. In Experiment 2, substantially more extinction bursts occurred following within-session transitions to extinction than between-sessions transitions. This suggests that the type of transition to extinction may influence response rates early on during extinction, and therefore the likelihood of an extinction burst. One potential explanation for this finding is differences in the stimuli present at the beginning of the first session of extinction across transition types. As the stimulus conditions normally paired with the beginning of each session were not present following the first within-session transition to extinction, the change in the schedule in effect may have been more discriminable.

This analysis would suggest that altering the discriminability of the transition from baseline to extinction may be an effective method for mitigating the intensity of a potential extinction burst or eliminating it altogether. To test this possibility, the discriminability of the transition from baseline to extinction and the consequent effect on response rates early in extinction might be investigated profitably in future experiments. The demonstration of a relation between these two variables across various methods for altering the discriminability of 


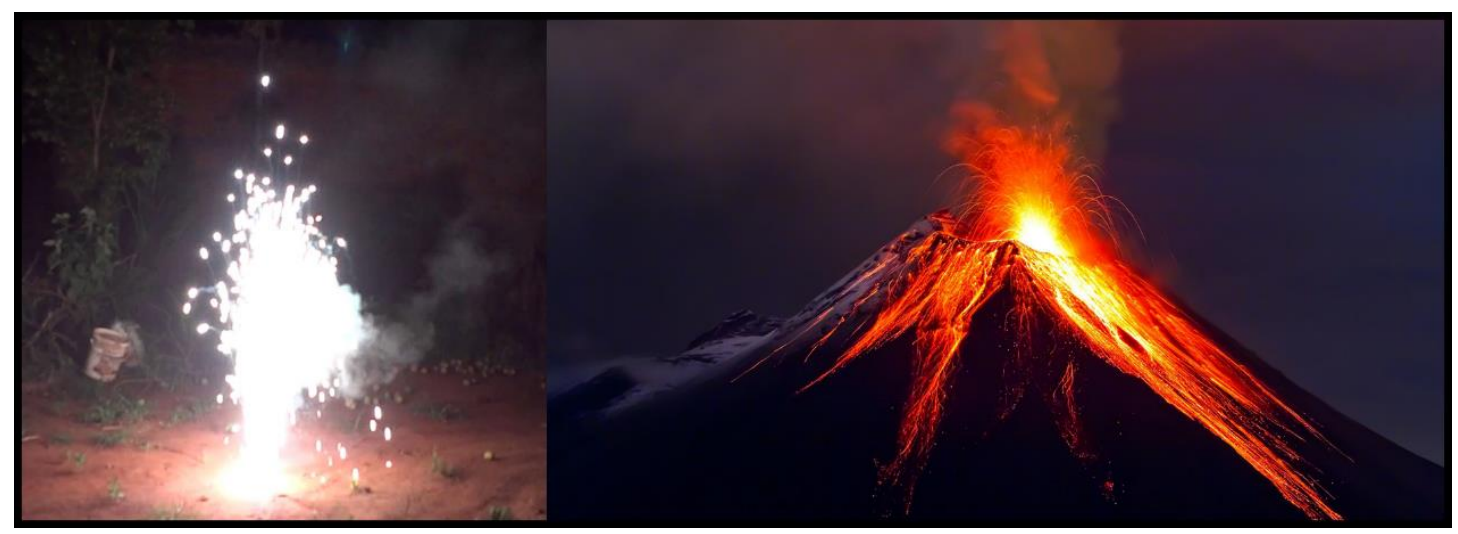

Figure 11. A comparison of an extinction burst (left panel) and an extinction eruption (right panel) obtained during two different transitions to extinction in Experiment 2. Note how much more intense the right panel looks. 
the transition to extinction would aid in further establishing the generality of the extinction burst. Potential methods for altering the discriminability of the onset of extinction aside from changing if transition occurs between- or within-session include adjusting the ratio requirement of the schedule in effect during baseline, changing the way extinction is implemented (i.e. eliminating reinforcer delivery or the response-reinforcer dependency), and comparing the presence and absence of discriminative stimuli across baseline and extinction sessions.

Schedule used during baseline. One of the aims of the current series of experiments was to develop a procedure that reliably induced extinction bursts across repeated exposures to extinction. Based on the present results, none of the schedules of reinforcement enacted during Conditioning Phase 2 sessions seem suited to producing this effect. Following an FR 1 schedule of reinforcement the extinction burst reliably occurred, but only during the first two exposures to the Extinction phase for experimentally-naïve pigeons. Starting with the third exposure, additional extinction bursts either were infrequent or did not occur depending on the type of analysis. Conversely, following both VR schedules, the extinction burst occurred across up to nine exposures to extinction, but was an intermittent, unreliable phenomenon.

Given these differences in bursting across each experiment, the schedule of conditioning in effect during baseline may be one of the controlling variables of the extinction burst. Additional support for this claim comes from how the mean magnitude of the extinction burst was substantially higher following FR 1 schedules than VR schedules. This difference in magnitude across different schedules of reinforcement may be the reason that most experiments that have reported bursts have used continuous reinforcement prior to the onset of extinction (e.g. Goh \& Iwata, 1994; Holton, 1961; Margulies, 1961, Zarcone et al., 1993). 
Based on these schedule-specific differences in the dimensions of the extinction burst, additional investigation of how other schedules of reinforcement influence the extinction burst are necessary. One potential route of investigation would be to determine whether the differences between the VR and FR 1 schedules described above were a consequence of the differences in the ratio size across the two schedules or the way reinforcers were delivered (i.e. variable or fixed). Furthermore, given that extinction bursts are most frequently reported following FR 1 schedules of conditioning, follow-up experiments to Experiment 3 would be worthwhile. Specifically, additional experiments comparing experimentally naïve and non-naïve pigeons could reveal whether prior exposure to the repeated-extinctions procedure was responsible for the differences in the occurrence of the extinction burst across pigeons in Experiment 3.

Post-reinforcement pause analysis. Only a single extinction burst occurred during Experiment 2 when the phenomenon was evaluated by comparing run rates from the first minute of extinction to run rates from either the first or last minute of Conditioning Phase 2. This suggests that the increases in response rate observed during extinction that have classically been referred to as extinction bursts are in fact artifacts of the absence of reinforcer deliveries during extinction. Because reinforcers are not delivered, PRPs do not occur. Therefore, response rate calculations produce comparatively higher response rates than those obtained during preceding baseline sessions, which are then interpreted as extinction bursts.

The absence of extinction bursts according to this method of analysis, combined with the relative infrequency of the extinction burst when assessed via comparisons of response rates, raises questions concerning past reports of the phenomenon. As prior investigations of the frequency of the extinction burst (e.g. Lerman \& Iwata, 1995; Lerman et al., 1999) do not 
specify whether it was assessed via changes in run rate, response rate, or the overall number of responses in a session, definitive statements about the frequency and nature of the phenomenon cannot be made.

Based on the present results, the use of either run rates or overall response rates in an evaluation of the extinction burst influence the frequency with which the phenomenon occurs. Thus, additional comparisons of these two methods of analysis are warranted to assess the extent of their differences. A useful follow-up to this experiment would be to evaluate the effects of repeated exposures to extinction following an FR 2 schedule of conditioning on responding. The use of an FR 2 schedule during baseline would address many of the limitations discussed for each of the present experiments. First, given that reinforcers are delivered on a fixed schedule, the point of contact with the extinction contingency, and thus which responses should be included in an analysis of bursting, is evident. Second, the use of a ratio requirement of two responses allows for a comparison of bursting evaluated using response rates and run rates, while simultaneously keeping the ratio requirement as close to that of an FR 1 as possible.

\section{Constraints on Generality}

In addition to the various unique aspects of each version of the repeated-extinctions procedure employed in the three reported experiments, other details of the procedure consistent across experiments constrain the generality of the present findings. One such limitation was the use of a non-data criterion (Sidman, 1961) for the duration of Conditioning Phase 2. Given the emphasis on developing a procedure to reliably induce extinction burst across repeated exposures to extinction, a non-data criterion was selected to focus on the effect of repeated transitions to extinction on responding. Despite this rationale, the fact that Conditioning Phase 2 sessions were not conducted until responding was stable likely contributed to the considerations regarding 
variability addressed above. As such, it may prove useful to investigate whether analyses of bursting across repeated exposures to extinction are any different if sessions of Conditioning Phase 2 are conducted until responding is mathematically stable at both the whole-session and first-minute levels of analysis.

Another constraint on generality concerns the effects of behavioral history and prior exposure to the repeated-extinctions procedure. As noted in the discussion for Experiment 3, the one pigeon with prior experience with the repeated-extinctions procedure did not exhibit any instances of bursting following transitions from an FR 1 schedule to extinction. Additional comparisons of experimentally naïve and non-naïve would be useful to assess the extent of the effects of prior exposure to the repeated-extinctions procedure on the extinction burst.

Furthermore, given that all three pigeons used in Experiment 2 had prior experience with the repeated-extinctions procedure during Experiment 1, a replication of Experiment 2 using experimentally-naïve pigeons would be useful to confirm the generality of the obtained results and evaluate the effects of prior experience with extinction on responding during subsequent transitions.

\section{Conclusions}

As noted in the literature review, Lerman and Iwata (1995, Lerman et al. 1999) found that across a combined 154 experiments and case studies, the extinction burst occurred between 24$39 \%$ of the time. Across all of the present experiments, however, extinction bursts occurred between $0 \%$ and $42 \%$ of the time, depending on how it was measured. Although the repeatedextinction procedure did not reliably produce extinction bursts across successive exposures to extinction, the results suggest several potential controlling variables of the phenomenon. Variations in how the procedure was implemented (i.e., between- or within-session onset of the 
first extinction session of a block) or how the extinction burst was measured (e.g. total session or first-minute response rates, with the latter compared to the first or last minute of the preceding baseline session) across the experiments influenced the number of bursts that occurred and their magnitude, demonstrating the importance of these variables in determining the extinction burst.

Although the eliminative effects of extinction are sensitive to any number of variables in effect in baseline conditions (e.g. Nevin, 1974; Reynolds, 1968), the effects of these variables on the extinction burst remain virtually completely uninvestigated. This has not prevented the making of broad claims about the likelihood and nature of the extinction burst. The phenomenon frequently is defined as a potential - indeed, highly likely - consequence of extinction, with no reference to how the conditions in effect during baseline or extinction sessions may modulate its occurrence (e.g. Cooper et al., 2007; Domjan \& Burkhard, 1982; Lerman \& Iwata, 1995; Lerman et al., 1999; Reynolds, 1968). Thus, despite the absence of experimental evidence, the extinction burst has been interpreted as relatively insensitive to changes in the variables outlined previously. As the results of the present series of experiments show, this assessment is incorrect. The probability and dimensions of the extinction burst are sensitive to a host of variables including the schedule in effect during baseline, the type of transition from baseline to extinction, and how the phenomenon is measured.

The present results have thus begun the task of filling in the missing blanks concerning the nature of the extinction burst that have persisted over the past 60 years. In addition to demonstrating the sensitivity of the burst to variables in effect in baseline conditions, it also identified potential controlling variables of the phenomenon that might be fruitfully explored. This investigation of the relation between baseline conditions and extinction bursts, the role of the type of transition to extinction, and the definition of the phenomenon has aided in the 
formation of a more complete picture of the extinction burst and the factors that influence its frequency and magnitude. 


\section{References}

Anger, D., \& Anger, K. (1976). Behavior changes during repeated eight-day extinctions. Journal of the Experimental Analysis of Behavior, 26, 181-190.

doi:10.1901/jeab.1976.26-181

Antonitis, J. J. (1951). Response variability in the white rat during conditioning, extinction, and reconditioning. Journal of Experimental Psychology, 42, 273-281. doi:10.1037/h0060407

Appel, J. B., \& Hiss, R. (1962). The discrimination of contingent from noncontingent reinforcement. Journal of Comparative and Physiological Psychology, 55, 37-39. doi:10.1037/h0044613

Azrin, N. H., Hutchinson, R. R., \& Hake, D. F. (1966). Extinction-induced aggression. Journal of the Experimental Analysis of Behavior, 9, 191-204.

doi:10.1901/jeab.1966.9-191

Cooper, J. O., Heron, T. E., \& Heward, W. L. (2007). Applied behavior analysis ( $2^{\text {nd }}$ ed.). Upper Saddle River, NJ: Pearson.

Domjan and Burkhard (1982). The principles of learning and behavior. Belmont, CA: Brooks/Cole.

Ducharme, J. M., \& Van Houten, R. (1994). Operant extinction in the treatment of severe maladaptive behavior. Behavior Modification, 18, 139-170. doi: $10.1177 / 01454455940182001$

Eckerman, D. A., \& Lanson, R. N. (1969). Variability of response location for pigeons responding under continuous reinforcement, intermittent reinforcement. Journal of the Experimental Analysis of Behavior, 12, 73-80. 
doi:10.1901/jeab.1969.12-73

Edwards, D. D., Peek, V., Wolfe, F. (1970). Independently delivered food decelerates fixed-ratio rates. Journal of the Experimental Analysis of Behavior, 14, 301-307. doi:10.1901/jeab.1970.14-301

Epstein, R. (1983). Resurgence of previously reinforced behavior during extinction. Behaviour Analysis Letters, 3, 391-397.

Ferster, C. B., \& Skinner, B. F. (1957). Schedules of reinforcement. New York, NY: AppletonCentury-Crofts. doi:10.1037/10627-000

Fleshler, M. \& Hoffman, H. S. (1962). A progression for generating variable-interval schedules. Journal of the Experimental Analysis of Behavior, 5, 529-530. doi:10.1901/jeab.1962.5-529

Gilbert, T. F. (1958). Fundamental dimensional properties of the operant. Psychological Review, $65,272-282$. doi:10.1037/h0044071

Goh, H. L., \& Iwata, B. A. (1994). Behavioral persistence and variability during extinction of self-injury maintained by escape. Journal of Applied Behavior Analysis, 27, 173-174. doi:10.1901/jaba.1994.27-173

Holton, R. B. (1961). Amplitude of an instrumental response following the cessation of reward. Child development, 32, 107-116. doi:10.2307/1126178

Kazdin, A. E. (1994). Behavior modification in applied settings ( $5^{\text {th }}$ ed.). Pacific Grove, CA: Brooks/Cole. 
Keller, F. S., \& Schoenfeld, W. N. (1950). Principles of psychology. New York, NY: AppletonCentury-Crofts.

Kelly, J. F., \& Hake, D. F. (1970). An extinction-induced increase in an aggressive response with humans. Journal of the Experimental Analysis of Behavior, 14, 153-164. doi:10.1901/jeab.1970.14-153

Lattal, K. A. (1972). Response-reinforcer independence and conventional extinction after fixedinterval and variable-interval schedules. Journal of the Experimental Analysis of Behavior, 18, 133-140.

doi:10.1901/jeab.1972.18-133

Lattal, K. A., \& Maxey, G. C. (1971). Some effects of response independent reinforcers in multiple schedules. Journal of the Experimental Analysis of Behavior, 16, 225-231. doi:10.1901/jeab.1971.16-225

Lattal, K. A., \& St. Peter Pipkin, C. (2009). Resurgence of previously reinforced responding: Research and application. The Behavior Analyst Today, 10, 254-265. doi:10.1037/h0100669

Lattal, K. A., St. Peter., C., \& Escobar, R. (2013). Operant extinction: elimination and generation of behavior. In G. J. Madden, W. V. Dube, T. D. Hackenberg, G. P. Hanley, \& K. A. Lattal (Eds.), APA handbook of behavior analysis, vol 2: translating principles into practice (pp. 77-107). Washington, DC: APA.

doi:10.1037/13938-004

Lerman, D. C., \& Iwata, B. A. (1995). Prevalence of the extinction burst and its attenuation during treatment. Journal of Applied Behavior Analysis, 28, 93-94. doi:10.1901/jaba.1995.28-93 
Lerman, D. C., \& Iwata, B. A. (1996). Developing a technology for the use of operant extinction in clinical settings: An examination of basic and applied research. Journal of Applied Behavior Analysis, 29, 345-385.

doi:10.1901/jaba.1996.29-345

Lerman, D. C., Iwata, B. A., \& Wallace, M. D. (1999). Side effects of extinction: Prevalence of bursting and aggression during the treatment of self-injurious behavior. Journal of Applied Behavior Analysis, 32, 1-8.

doi:10.1901/jaba.1999.32-1

Mace, F. C., McComas, J. J., Mauro, B. C., Progar, P. R., Taylor, B., Ervin, R., \& Zangrillo, A. N. (2010). Differential reinforcement of alternative behavior increases resistance to extinction: clinical demonstration, animal modeling, and clinical test of one solution. Journal of the Experimental Analysis of Behavior, 93, 349-367.

doi:10.1901/jeab.2010.93-349

Margulies, S. (1961). Response duration in operant level, regular reinforcement and extinction. Journal of the Experimental Analysis of Behavior, 4, 317-321. doi:10.1901/jeab.1961.4-317

Martin, G., \& Pear, J. (1992). Behavior modification: What it is and how to do it (4 ${ }^{\text {th }}$ ed.). Englewood Cliffs, NJ: Prentice Hall.

Mechner, F. (1958). Sequential dependencies of the lengths of consecutive response runs. Journal of the Experimental Analysis of Behavior, 1, 229-233. doi:10.1901/jeab.1958.1-229

Millenson, J. R. (1967). Principles of behavioral analysis. New York: MacMillan. 
Nevin, J. A. (1974). Response strength in multiple schedules. Journal of the Experimental Analysis of Behavior, 21, 389-408.

doi:10.1901/jeab.1974.21-389

Notterman, J. M., \& Mintz, D. E. (1965). Dynamics of response. New York, NY: Wiley.

Rescorla, R. A., \& Skucy, J. C. (1969). Effect of response independent reinforcers during extinction. Journal of Comparative and Physiological Psychology, 67, 381-389. doi:10.1037/h0026793

Reynolds, G. S. (1968). A primer of operant conditioning. New York: Scott Foresman.

Roper, T. J. (1981). What is meant by the term "schedule-induced" and how general is schedule induction? Animal Learning and Behavior, 9, 433-440. doi:10.3758/BF03209773

Sajwaj, T., Twardosz, S., \& Burke, M. (1972). Side effects of extinction procedures in a remedial preschool. Journal of Applied Behavior Analysis, 5, 163-175. doi:10.1901/jaba.1972.5-163

Sidman, M. (1960). Tactics of scientific research. Authors Cooperative, Inc.

Skinner, B. F. (1938). Behavior of organisms. New York, NY: Appleton-Century-Crofts. Skinner, B. F. (1956). A case history in scientific method. American Psychologist, 11, 221-233. Reprinted in B. F. Skinner (1961), Cumulative Record, (pp. 76-99). New York: Appleton-Century-Crofts.

Staddon, J. E. R. (1977). Schedule-induced behavior. In W. K. Honig \& J. E. R. Staddon (Eds.), Handbook of operant behavior (pp. 125-152). Englewood Cliffs, NJ: Prentice-Hall.

Stokes, P. D. (1995). Learned variability. Animal Learning \& Behavior, 23, 164-176. doi:10.3758/bf03199931 
Terrace, H. S. (1963). Discrimination learning with and without "errors." Journal of the Experimental Analysis of Behavior, 6, 1-27. doi:10.1901/jeab.1963.6-1

Thompson, T., \& Bloom, W. (1966). Aggressive behavior and extinction-induced response-rate increase. Psychonomic Science, 5, 335-336.

doi:10.3758/bf03328426

Ward, R. D., \& Odum, A. L. (2008). Sensitivity of conditional-discrimination performance to within-session variation of reinforcer frequency. Journal of the Experimental Analysis of Behavior, 90, 301-311.

doi:10.1901/jeab.2008.90-301

Zarcone, J. R., Iwata, B. A., Vollmer, T. R., Jagtiani, S., Smith, R. G., \& Mazaleski, J. L. (1993). Extinction of self-injurious escape behavior with and without instructional fading. Journal of Applied Behavior Analysis, 26, 353-360.

doi:10.1901/jaba.1993.26-353

Zeiler, M. D. (1968). Fixed and variable schedules of response-independent reinforcement. Journal of the Experimental Analysis of Behavior, 11, 405-414. doi:10.1901/jeab.1968.11-405 